Is the Potential for International Diversi cation Disappearing? A Dynamic Copula Approach

Peter Christoffersen, Vihang Errunza, Kris Jacobs and Hugues Langlois

CREATES Research Paper 2012-48 


\title{
Is the Potential for International Diversification Disappearing? A Dynamic Copula Approach*
}

\author{
Peter Christoffersen Vihang Errunza Kris Jacobs Hugues Langlois \\ University of Toronto McGill University University of Houston McGill University \\ May 24, 2012
}

\begin{abstract}
International equity markets are characterized by nonlinear dependence and asymmetries. We propose a new dynamic asymmetric copula model to capture long-run and short-run dependence, multivariate nonnormality, and asymmetries in large cross-sections. We find that copula correlations have increased markedly in both developed markets (DMs) and emerging markets (EMs), but they are much lower for EMs than for DMs. Tail dependence has also increased but its level is still relatively low for EMs. We propose new measures of dynamic diversification benefits that take into account higher order moments and nonlinear dependence. The benefits from international diversification have reduced over time, drastically so for DMs. EMs still offer significant diversification benefits, especially during large market downturns.
\end{abstract}

JEL Classification: G12

Keywords: Asset allocation, dynamic dependence, dynamic copula, asymmetric dependence.

\footnotetext{
${ }^{*}$ Christoffersen, Errunza, and Jacobs gratefully acknowledge financial support from IFM2 and SSHRC. Errunza is also supported by the Bank of Montreal Chair at McGill University and Jacobs by the Bauer Chair at the University of Houston. Hugues Langlois is funded by NSERC, CIREQ and IFM2. We are grateful to the Editor, Geert Bekaert, as well as two anonymous referees for comments on an earlier version of the paper. We also thank Lieven Baele, Greg Bauer, Phelim Boyle, Ines Chaieb, Rob Engle, Frank de Jong, Rene Garcia, Sergei Sarkissian, Ernst Schaumburg, and seminar participants at the Bank of Canada, EDHEC, HEC Montreal, NYU Stern, SUNY Buffalo, Tilburg University, and WLU for helpful comments.
} 


\section{Introduction}

Understanding and quantifying the evolution of security co-movements is critical for asset pricing and portfolio allocation. The co-movements between equity markets in different countries determine how the diversification benefits of international investing have evolved over time. Measuring these benefits requires an answer to three distinct questions.

First, how has cross-country dependence changed through time? Most of the available evidence on the time-variation in cross-country correlations is based on factor models. ${ }^{1}$ Bekaert, Hodrick, and Zhang (2009) convincingly argue that the evidence from this literature is mixed at best and state that (see p. 2591): "It is fair to say that there is no definitive evidence that cross-country correlations are significantly and permanently higher now than they were, say, ten years ago." They investigate international stock return co-movements for 23 DMs during 1980-2005, and find an upward trend in return correlations only among the subsample of European stock markets.

Second, is correlation a satisfactory measure of dependence in international markets, or do we need to consider different measures, notably those that focus on the dependence between tail events? This question is related to the analysis of correlation asymmetries, and changes in correlation as a function of business cycle conditions or stock market performance. Following Longin and Solnik (2001), Ang and Bekaert (2002) and Ang and Chen (2002), the hypothesis that cross-market correlations rise in periods of high volatility has been supplanted by the notion that correlations increase in down markets, but not in up markets. Longin and Solnik (2001) use extreme value theory in bivariate monthly models for the U.S. with either the U.K., France, Germany, or Japan during 1959-1996. Ang and Bekaert (2002) develop a regime switching dynamic asset allocation model, and estimate it for the U.S., U.K., and Germany over the period 1970-1997. Both papers estimate return extremes at predetermined threshold values, i.e. they define the tail observations ex ante, and then compute unconditional correlations for the tail for the developed markets above.

Third, over the last two decades much of the focus in international finance has shifted to the diversification benefits offered by emerging markets. ${ }^{2}$ Hence, it is important to investigate whether

\footnotetext{
${ }^{1}$ King, Sentana, and Wadhwani (1994) do not find evidence of increasing cross-country correlations for 16 developed markets during the period 1970-1988, except around the market crash of 1987. Carrieri, Errunza, and Hogan (2007) do not find a common pattern in the correlation trend for eight emerging markets (EMs) during 1977-2000. Eiling and Gerard (2007) find an upward time trend in co-movements between 24 developed markets but not between 26 emerging markets over the period 1973-2005. Goetzmann, Li, and Rouwenhorst (2005) document substantial changes in the correlation structure of world equity markets over the past 150 years. Baele and Inghelbrecht (2009) report increasing correlations over the period 1973-2007 for their sample of 21 DMs. See also Karolyi and Stulz (1996), Forbes and Rigobon (2002), Brooks and Del Negro (2003), Lewis (2006), and Rangel (2011).

${ }^{2}$ For early studies documenting the benefits of international diversification, see Solnik (1974) for developed markets and Errunza (1977) for emerging markets. For more recent evidence, see for example Erb, Harvey and Viskanta (1994), DeSantis and Gerard (1997), Errunza, Hogan and Hung (1999), and Bekaert and Harvey (2000).
} 
there are meaningful differences between emerging markets (EMs) and developed markets (DMs) in cross-country dependence and tail dependence. Existing studies analyze tail dependence for a few DMs, and there is limited evidence on time variation in tail dependence. Moreover, with some exceptions, most notably the paper by Bekaert, Hodrick, and Zhang (2009), there is little regional analysis.

In this paper, we provide a comprehensive empirical study of the dynamic evolution of dependence and tail dependence for a large set of developed and emerging markets, as well as for regional subsets. We offer two methodological contributions. First, to accommodate well-documented empirical regularities in the literature, we propose a new model that allows for asymmetries, trends in dependence, and deviations from multivariate normality. To capture these stylized facts, we generalize the flexible dynamic conditional correlation (DCC) model of Engle (2002) and Tse and Tsui (2002) in two ways: First, we do not model dependence as mean-reverting but instead allow it to mean revert to a possibly nonlinear trend. Second, we do not model linear correlations, which are only sufficient under multivariate normality, instead we model the joint distribution using time-varying copulas to capture nonlinear dependence across markets. Our second methodological contribution is the development of a dynamic measure of diversification benefits that takes into account higher order moments and non-linear dependence. We also analyze this measure under the special case of multivariate normal returns.

We develop a novel dynamic asymmetric copula (DAC) model which allows for asymmetric and dynamic tail dependence in large portfolios. We implement this model relying on recent econometric innovations that overcome dimensionality problems, and that facilitate estimation using large numbers of countries and long time series. Specifically, we rely on the numerically efficient composite likelihood procedure proposed by Engle, Shephard, and Sheppard (2008). The composite likelihood estimation procedure is essential for estimating dynamic dependence models on international equity data with large cross-sections and long time series.

Using our new framework, we characterize time-varying dependence using weekly returns during the 1973-2009 period for a large number of countries (either thirteen or seventeen EMs, sixteen DMs, as well as combinations of the EM and DM samples). We also provide evidence on threshold correlations and other indicators of asymmetric tail dependence. Our implementation is relatively straightforward and computationally fast. We thus demonstrate that it is possible to estimate dependence patterns in international markets using large numbers of countries and extensive time series. We extend existing results on dependence to a more recent period characterized by significant liberalizations for the EM sample, as well as substantial market turmoil during 2007-2009, which helps identify tail dependence. We obtain the following findings.

First and foremost, we find extremely robust evidence that international dependence between 
stock markets, as measured by copula correlations, has been significantly trending upward for both DMs and EMs. However, the dependence between DMs has been higher than the dependence between EMs at all times in our sample. For developed markets, the average dependence with other developed markets is higher than the average dependence with emerging markets. For emerging markets, the dependence with developed markets is generally somewhat higher than the dependence with other emerging markets, but the differences are small. When dividing our sample into four regions: EU, developed non-EU, Latin America, and Emerging Eurasia, we find that the dependence between all four regions has gone up, and so has the average dependence within each region. While the range of dependence for DMs has narrowed around the increasing trend in dependence levels, this is not the case for EMs.

Second, we find overwhelming evidence that the assumption of multivariate normality is inappropriate, which is consistent with the existing literature. Our parameter estimates for the dynamic copula models indicate substantial tail dependence, which furthermore appears to be asymmetric and increasing through time for both EMs and DMs.

Third, the most striking finding regarding tail dependence is that the level of tail dependence is still very low at the end of the sample period for EMs as compared to DMs. Our findings on tail dependence thus suggest that EMs offer diversification benefits during large market moves. The underlying intuition for this finding is that while financial crises in EMs are frequent, many of them are country-specific. Our new diversification measure that takes into account the time-variation in dependence and nonnormalities present in the data indicates that EMs provide better diversification potential than DMs. Thus, although the benefits of international diversification might have lessened over time both for DMs and EMs, a strong case can still be made for EMs. Indeed, the diversification benefits of adding emerging markets to a portfolio appear to be significant.

Fourth, we demonstrate that the new DAC model can capture the empirical asymmetries in threshold correlations. We document asymmetric threshold correlation patterns for EMs, and find that they differ from those for DMs. Longin and Solnik (2001) and Ang and Bekaert (2002) document asymmetric threshold correlation patterns among a select group of major developed markets, but to the best of our knowledge the literature does not contain evidence on EMs. We demonstrate that our multivariate asymmetric model can capture the threshold correlation patterns observed in DMs and EMs.

Fifth, we use a regression analysis to link the time variation in dependence to economic fundamentals, market characteristics, and measures of financial openness. We also investigate the relationship between dependence and volatility. Our model does not assume a factor structure but we do find a significant positive association between copula correlations and volatilities. We find that neither volatility nor other financial and macro variables are able to drive out the trend in 
copula correlations.

The paper proceeds as follows. Section 2 introduces the new DAC model with dynamic copula correlations, allowing for dynamic tail dependence and asymmetries. We place special emphasis on the estimation of this model for large systems. Section 3 presents the data, as well as the empirical results on time variation in copula correlations. Section 4 introduces a new non-linear conditional measure of diversification benefits that can take into account the nonlinear dependence, asymmetries, and nonnormalities in the DAC model. We also discuss empirical estimates of this measure. Section 5 discusses additional economic implications, including tail dependence, threshold correlations, and dependence over longer horizons. Section 5 also contains a regression analysis of the economic determinants of the dependence measures. Section 6 concludes.

\section{Dynamic Dependence Models for Many Equity Markets}

This section outlines the general model we use to capture dynamic dependence across equity markets. Our dynamic copula approach allows for multivariate nonnormalities, and models copula correlations as reverting back to a long-run mean which consists of a constant as well as a timevarying part. This model feature is critical to capture dependencies that are potentially trending over time. We also describe how this model can be reliably estimated using a large cross-section of assets.

\subsection{The Dynamic Conditional Copula Approach}

Our objective is to characterize dependence in a general way using the largest possible cross-section of international equity markets. In the existing literature, implementations of multivariate GARCH models have traditionally used a limited number of countries because of dimensionality problems. ${ }^{3}$ Recent modeling innovations by Engle (2002) and Tse and Tsui (2002), combined with implementation techniques discussed in Section 2.5 below, make it possible to study larger cross-sections of countries.

However, in characterizing international stock market dependence, a crucial issue is the use of the multivariate normal distribution which is usually relied upon to implement dynamic correlation models. The multivariate normal distribution is the standard choice in the literature because it is convenient, and because quasi maximum likelihood results ensure that the dynamic correlation parameters will be estimated consistently even when the normal distribution assumption is incorrect,

\footnotetext{
${ }^{3}$ See Kroner and $\mathrm{Ng}$ (1998) and Solnik and Roulet (2000) for a more elaborate discussion of the restrictions imposed in the first generation of multivariate GARCH models.
} 
as long as the dynamic models are correctly specified.

While the multivariate normal distribution is a convenient statistical choice, the economic motivation for using it is more dubious. It is well-known (see for example Longin and Solnik (2001), and Ang and Bekaert (2002)) that international equity returns display threshold correlations not captured by the normal distribution: Large down moves in international equity markets are highly correlated, which is of course crucial for assessing the benefits of diversification. The dynamic correlation models of Engle (2002) can generate more realistic threshold correlations, but likely not to the degree required by the data. Moreover, they are symmetric by design, and cannot accommodate Longin and Solnik's (2001) finding that returns are more correlated in down markets. We therefore go beyond the dynamic multivariate normal distributions used in Engle (2002) and Tse and Tsui (2002) and introduce dynamic copula models which have the potential to generate empirically relevant levels of threshold correlations, as well as asymmetric threshold correlations.

Copulas constitute an extremely convenient tool for building a multivariate distribution for a set of assets from any choice of marginal distributions for each individual asset. ${ }^{4}$ From Patton (2006), who relies on Sklar (1959), we can decompose the conditional multivariate density function of a vector of returns for $N$ countries, $f_{t}\left(R_{t}\right)$, into a conditional copula density function, $c_{t}$, and the product of the conditional marginal distributions $f_{i, t}\left(R_{i, t}\right)$

$$
f_{t}\left(R_{t}\right)=c_{t}\left(F_{1, t}\left(R_{1, t}\right), F_{2, t}\left(R_{2, t}\right), \ldots, F_{N, t}\left(R_{N, t}\right)\right) \prod_{i=1}^{N} f_{i, t}\left(R_{i, t}\right)
$$

where $R_{t}$ is a vector of returns at time $t$ comprised of individual returns $R_{1, t}$ to $R_{N, t}$, and $F_{i, t}$ is the cumulative distribution function of $R_{i, t}$.

From this the multivariate log-likelihood function can be constructed as

$$
L=\sum_{t=1}^{T} \sum_{i=1}^{N} \log \left(f_{i, t}\left(R_{i, t}\right)\right)+\sum_{t=1}^{T} \log \left(c_{t}\left(F_{1, t}\left(R_{1, t}\right), F_{2, t}\left(R_{2, t}\right), \ldots, F_{N, t}\left(R_{N, t}\right)\right)\right)
$$

The upshot of this decomposition is that we can make assumptions about the marginal densities that are independent of the assumptions made about the copula function. We now discuss the modeling of the marginal densities, and subsequently we address how copula techniques can capture asymmetric threshold correlations.

\footnotetext{
${ }^{4}$ McNeil, Frey and Embrechts (2005) provide an authoritative review of the use of constant copulas in risk management.
} 


\subsection{Modeling the Marginal Density}

For modeling the marginal density, the critical issues are dynamic volatility and the modeling of asymmetries. Asymmetries are traditionally modeled through the leverage effect, which has been found to be an important stylized fact in equity index returns. The leverage effect is an asymmetric volatility response that captures the fact that a large negative shock to an equity market increases the equity market volatility by much more than a positive shock of the same magnitude. See for example Black (1976) and Engle and Ng (1993). See Bekaert and Wu (2000) for evidence on international markets.

Existing studies usually find that even when including a leverage effect, model residuals contain evidence of remaining skewness and fat tails. We therefore allow for asymmetry in the marginal return distribution by modeling a leverage effect, but also by using an asymmetric marginal distribution for the return innovation in each country. To capture both effects as well as volatility persistence and heteroskedasticity, we assume that the return on asset $i$ at time $t$ follows an Engle and Ng (1993) dynamic

$$
\begin{aligned}
R_{i, t} & =\mu_{i, t}+\varepsilon_{i, t}=\mu_{i, t}+\sigma_{i, t} z_{i, t} \\
\sigma_{i, t}^{2} & =\omega_{i}+\alpha_{i}\left(\varepsilon_{i, t-1}-\gamma_{i} \sigma_{i, t-1}\right)^{2}+\beta_{i} \sigma_{i, t-1}^{2} .
\end{aligned}
$$

We assume that the distributions of the innovations differ across assets, but are constant over time and follow the skewed $t$ distribution of Hansen (1994) which is detailed in Appendix A. Here we simply denote it by $F_{i, t}\left(z_{i, t}\right)=F_{\chi_{i}, \varrho_{i}}\left(z_{i, t}\right) .{ }^{5}$ Country $i$ 's residual conditional skewness is driven by the parameter $\chi_{i}$ and its degree of conditional kurtosis is controlled by the parameter $\varrho_{i}$. We write the cumulative distribution function as

$$
\eta_{i, t}=F_{\chi_{i}, \varrho_{i}}\left(z_{i, t}\right)
$$

Note that in our approach the individual return shock distributions are constant through time but the individual return distributions do vary through time because the return mean and variance are dynamic.

\footnotetext{
${ }^{5}$ Alternatively, one can also use a nonparametric approach for modeling the marginals in copula modeling, see for example Chen and Fan (2006). We use Hansen's (1994) skewed $t$ distribution to ensure that the copula-based multivariate distribution will be well specified, which allows us to conduct statistical inference, relying on the asymptotic theory discussed in Engle, Shephard and Sheppard (2008) which requires a parametric approach. We verified that our parameter estimates are similar to semiparametric estimates that rely on combining the empirical distribution function with a Generalized Pareto distribution for the tails of the distribution. See McNeil (1999) and McNeil and Frey (2000) for more detail on this approach.
} 


\subsection{Allowing for Multivariate Nonnormality and Asymmetry}

A useful model of international equity returns needs to account for tail dependence and asymmetries in threshold correlations mentioned in Section 2.1, which are well-established empirical facts. The asymmetries discussed in Section 2.2 only address asymmetries in the marginal densities, and not the well-known multivariate asymmetries and asymmetric threshold correlations. We rely on copula models to capture these multivariate asymmetries.

Within the class of copula models, the most widely applied copula function is based on the multivariate normal distribution and referred to as the normal copula. Though convenient to use, it is not flexible enough to capture the tail dependence in asset returns. While allowing for tail dependence, the more flexible $t$ copula unfortunately implies symmetric threshold correlations. Asymmetry in the bivariate distribution of asset returns has generally been modeled using copulas from the Archimedean family which include the Clayton, the Gumbel, and the Joe-Clayton specifications. ${ }^{6}$ However, these models are not easily generalized to high-dimensional applications.

We therefore consider the skewed $t$ distribution discussed in Demarta and McNeil (2004) and use the implied skewed $t$ copula whose cumulative distribution function, $C_{t}$, is given by

$$
C_{t}\left(\eta_{1, t}, \eta_{2, t}, \ldots, \eta_{N, t} ; \Psi, \lambda, \nu\right)=t_{\Psi, \lambda, \nu}\left(t_{\lambda, \nu}^{-1}\left(\eta_{1, t}\right), t_{\lambda, \nu}^{-1}\left(\eta_{2, t}\right), \ldots, t_{\lambda, \nu}^{-1}\left(\eta_{N, t}\right)\right)
$$

where $\lambda$ is an asymmetry parameter, $\nu$ is the degree of freedom parameter, $t_{\Psi, \lambda, \nu}$ is the multivariate skewed Student's $t$ density with correlation matrix $\Psi$, and $t_{\lambda, \nu}^{-1}$ is the inverse cumulative distribution function of the univariate skewed $t$ distribution.

Note that the copula correlation matrix $\Psi$ is defined by the correlation of the copula shocks $z_{i, t}^{*} \equiv t_{\lambda, \nu}^{-1}\left(\eta_{i, t}\right)$ and not of the return shocks $z_{i, t}$. Notice also that

$$
z_{i, t}^{*} \equiv t_{\lambda, \nu}^{-1}\left(\eta_{i, t}\right)=t_{\lambda, \nu}^{-1}\left(F_{\chi_{i}, \varrho_{i}}\left(z_{i, t}\right)\right)
$$

so that if the marginal distributions $F_{\chi_{i}, \delta_{i}}$ are close to the $t_{\lambda, \nu}$ distribution, then $z_{i, t}^{*}$ will be close to $z_{i, t}$. The skewed $t$ copula is built from the skewed multivariate $t$ distribution and the symmetric $t$ copula is nested when $\lambda$ tends to zero. If the degree of freedom tends to infinity, the normal copula is obtained. Appendix A provides the details needed to implement the skewed $t$ copula. Note that the marginal model in Section 2.2 captures any univariate skewness present in the equity returns. The $\lambda$ parameter captures multivariate asymmetry.

The skewed $t$ copula is parsimonious, tractable in high dimension, and flexible, allowing us to model non-linear and asymmetric dependence with the degree of freedom parameter, $\nu$, and the

\footnotetext{
${ }^{6}$ See for example Patton $(2004,2006)$.
} 
asymmetry parameter, $\lambda$, while retaining a copula correlation matrix, $\Psi$, which can be made timevarying as we will see in the next section. Figure A.1 plots probability contours for the bivariate case for two parameterizations of the skewed $t$ copula, as well as the special cases of the $t$ copula and the normal copula. The probability levels for each contour are kept the same for all four figures. The ability of the skewed $t$ copula to generate substantial asymmetries with realistic parameter values is evident. For the sake of parsimony in our high-dimensional applications, we report on a version of the skewed $t$ copula where the asymmetry parameter $\lambda$ is a scalar. It is straightforward to develop a more general version of the skewed $t$ copula allowing for an $N$-dimensional vector of asymmetry parameters, but it is difficult to estimate such a model on a large number of countries.

\subsection{Dynamic Copula Correlations}

We now build on the linear correlation techniques developed by Engle (2002) and Tse and Tsui (2002) to model dynamic copula correlations. As in the standard dynamic conditional correlation (DCC) model, dynamic correlations are driven by a multivariate GARCH process. However, the copula shocks $z_{i, t}^{*} \equiv t_{\lambda, \nu}^{-1}\left(\eta_{i, t}\right)$ are used as the model's building block instead of the return shocks $z_{i, t}{ }^{7}$

The copula correlation dynamic is driven by the matrix $\Upsilon_{t}$ and the cross-products of the return shocks

$$
\Gamma_{t}=\left(1-\beta_{\Gamma}-\alpha_{\Gamma}\right)\left[\left(1-\varphi_{\Gamma}\right) \Omega+\varphi_{\Gamma} \Upsilon_{t}\right]+\beta_{\Gamma} \Gamma_{t-1}+\alpha_{\Gamma} \bar{z}_{t-1}^{*} \bar{z}_{t-1}^{* \top}
$$

where $\bar{z}_{i, t}^{*}=z_{i, t}^{*} \sqrt{\Gamma_{i i, t}}$ using the Aielli (2009) modification, $\beta_{\Gamma}, \alpha_{\Gamma}$, and $\varphi_{\Gamma}$ are scalars, and $\bar{z}_{t}^{*}$ is an $N$-dimensional vector with typical element $\bar{z}_{i, t}^{*}$. The conditional copula correlations are defined via the normalization

$$
\Psi_{i j, t}=\Gamma_{i j, t} / \sqrt{\Gamma_{i i, t} \Gamma_{j j, t}} .
$$

This normalization ensures that all copula correlations remain in the -1 to 1 interval.

Note that the dynamic conditional copula correlation matrix mean-reverts at time $t$ to a weighted average of a constant $\Omega$ and a time-varying matrix $\Upsilon_{t}$ with weighting parameter $\varphi_{\Gamma}$. We refer to this model as the dynamic asymmetric copula (DAC) model. Its components are as follows.

First, $\Omega$ is a constant copula correlation matrix. Therefore, by setting $\varphi_{\Gamma}$ to 0 we obtain the DCC approach of Engle (2002) as applied to copula correlations. Second, the matrix $\Upsilon_{t}$ contains information about time trends and explanatory variables. While this matrix can take on a very general form, as described in Appendix B, we consider here a simplified version with only a time

\footnotetext{
${ }^{7}$ Unless one uses the normal copula, the fractiles do not have zero mean and unit variance. We therefore standardize the $z_{i}^{*}$ in the dynamic copula dependence model.
} 
trend. For simplicity, we further constrain the time trend parameters to be equal across all developed markets and also across all emerging markets. The resulting matrix $\Upsilon_{t}$ is given by

$$
\Upsilon_{t}=\left[\begin{array}{cc}
\Upsilon_{D M, t} & \Upsilon_{D M, E M, t} \\
\Upsilon_{D M, E M, t}^{\top} & \Upsilon_{E M, t}
\end{array}\right]
$$

where $\Upsilon_{D M, t}$ is a $N_{D M} \mathrm{x} N_{D M}$ correlation matrix with all off-diagonal elements equal to $\frac{\delta_{D M}^{2} t^{2}}{1+\delta_{D M}^{2} t^{2}}$, $\Upsilon_{E M, t}$ is a $N_{E M} \mathrm{x} N_{E M}$ correlation matrix with all off-diagonal elements equal to $\frac{\delta_{E M}^{2} t^{2}}{1+\delta_{E M}^{2} t^{2}}$, and $\Upsilon_{D M, E M, t}$ is a $N_{D M} \mathrm{x} N_{E M}$ matrix with all elements equal to $\frac{\delta_{D M} \delta_{E M} t^{2}}{1+\delta_{D M} \delta_{E M} t^{2}}$, where $\delta_{D M}$ and $\delta_{E M}$ are parameters to be estimated. ${ }^{8}$

The conditional copula correlation matrix can be seen as the weighted average of a slowly varying component, $\left(1-\varphi_{\Gamma}\right) \Omega+\varphi_{\Gamma} \Upsilon_{t}$, the lagged conditional correlation matrix, $\Gamma_{t-1}$, and the lagged crossproduct of the standardized copula shocks. Note that a negative pair of copula shocks impact correlation in the same way as do a positive pair of copula shocks of the same magnitude. We also investigated an alternative specification using the cross-products of the re-centered copula shocks, $\left(\bar{z}_{t-1}^{*}-\xi_{\Gamma} \iota\right)\left(\bar{z}_{t-1}^{*}-\xi_{\Gamma} \iota\right)^{\top}$, where $\xi_{\Gamma}$ is a scalar and $\iota$ is an N-dimensional vector of ones. This specification introduces additional multivariate asymmetry into the model. However, our results showed that the estimates of parameter $\xi_{\Gamma}$ are not statistically significant, and the more general model does not yield a better fit than the model in (2.4). We therefore conclude that the skewed $t$ distribution adequately captures the multivariate asymmetries in the data.

\subsection{Estimation}

If $N$ denotes the number of equity markets under study, then the DAC model has $N(N-1) / 2+8$ parameters to be estimated. Below we will study up to 17 emerging markets and 16 developed markets, thus $N=33$ and so the DAC model will have 536 parameters. It is well recognized in the literature that it is impossible to estimate so many parameters reliably using numerical optimization techniques. ${ }^{9}$ In order to operationalize estimation, we use the average level of the copula correlations over the entire sample to fix the time-invariant parameters

$$
\Omega=\frac{\frac{1}{T} \sum_{t=1}^{T} \bar{z}_{t}^{*} \bar{z}_{t}^{* \top}-\varphi_{\Gamma} \frac{1}{T} \sum_{t=1}^{T} \Upsilon_{t}}{1-\varphi_{\Gamma}}
$$

\footnotetext{
${ }^{8}$ The parameterization of our trend correlation matrix is motivated by the approach in Marsaglia and Olkin (1984).

${ }^{9}$ See for instance Solnik and Roulet (2000) for a discussion.
} 
The numerical optimizer now only has to search in eight dimensions corresponding to the parameter vector $\theta=\left(\alpha_{\Gamma}, \beta_{\Gamma}, \varphi_{\Gamma}, \xi_{\Gamma}, \delta_{D M}, \delta_{E M}, \nu, \lambda\right)^{\top}$, rather than in the original 536 dimensions. Note that this implementation also ensures that the estimated DAC model yields a positive semi-definite correlation matrix, because $\bar{z}_{t}^{*} \bar{z}_{t}^{* \top}$ and thus $\Gamma$ is positive semi-definite by construction. Appendix $\mathrm{C}$ contains more details on the estimation of $\Omega$ in the DAC model.

Even in parsimonious models estimation is cumbersome with many assets due to the need to invert the $N$ by $N$ correlation matrix, $\Psi_{t}$, for every observation in the sample. Furthermore, the likelihood must be evaluated many times in the numerical optimization routine. More importantly, Engle, Shephard and Sheppard (2008) find that in large-scale estimation problems, the parameters $\alpha_{\Gamma}$ and $\beta_{\Gamma}$ which drive the correlation dynamics are estimated with bias when using conventional estimation techniques. They propose an ingenious solution based on the composite likelihood which, in our context, is defined as

$$
C L(\theta)=\sum_{t=1}^{T} \sum_{i=1}^{N} \sum_{j>i} \ln c_{t}\left(\eta_{i, t}, \eta_{j, t} ; \theta\right)
$$

where $c_{t}\left(\eta_{i, t}, \eta_{j, t} ; \theta\right)$ denotes the bivariate copula distribution of asset pair $i$ and $j$.

The composite log-likelihood is thus based on summing the log-likelihoods of pairs of assets. Each pair yields a valid (but inefficient) likelihood for $\theta$, but summing over all pairs produces an estimator which is relatively efficient, numerically fast, and free of bias even in large-scale problems. We use the composite log-likelihood in all our estimations below. We have found it to be very

reliable and robust, effectively turning a numerically impossible task into a manageable one. The composite likelihood procedure allows us to estimate dynamic copula correlations in larger systems of international equity data using longer time series of returns than previously done in the literature. This is important because long time series on large sets of countries are needed for the identification of variance and covariance dynamics.

\section{Empirical Dependence Analysis}

This section contains our empirical findings on dependence patterns. We first describe the different data sets and briefly discuss the univariate results. We then analyze the time-variation in copula correlations and dispersion in copula correlations across pairs of assets at each point in time and check if this dispersion has changed over time. 


\subsection{Data and Univariate Models}

We employ the following three data sets:

First, from DataStream we collect weekly closing U.S. dollar returns for the following 16 developed markets: Australia, Austria, Belgium, Canada, Denmark, France, Germany, Hong Kong, Ireland, Italy, Japan, Netherlands, Singapore, Switzerland, U.K., and U.S. This data set contains 1,901 weekly observations from January 12, 1973 through June 12, 2009.

Second, from Standard and Poor's we collect the IFCG weekly closing U.S. dollar returns for the following 13 emerging markets: Argentina, Brazil, Chile, Colombia, India, Jordan, Korea, Malaysia, Mexico, Philippines, Taiwan, Thailand, and Turkey. This data set contains 1,021 weekly observations from January 6, 1989 through July 25, 2008.

Third, from Standard and Poor's we collect the weekly closing investable IFCI U.S. dollar returns for the following 17 emerging markets: Argentina, Brazil, Chile, China, Hungary, India, Indonesia, Korea, Malaysia, Mexico, Peru, Philippines, Poland, South Africa, Taiwan, Thailand, and Turkey. This data set contains 728 weekly returns from July 7, 1995 through June 12, 2009.

We use two emerging markets data sets because each has their distinct advantages. The IFCG data set spans a longer time period, and represents a broad measure of emerging market returns, but is not available after July 25, 2008. The IFCI data set tracks returns on a portfolio of emerging market securities that are legally and practically available to foreign investors. The index construction takes into account portfolio flow restrictions, liquidity, size and float. It continues to be updated but the sample period is shorter, which is a disadvantage in model estimation and of course in assessing long-term trends in correlation.

Table 1 contains descriptive statistics on the 1989-2008 data set. While the cross-country variations are large, Table 1 shows that the average annualized return in the developed markets was $12.06 \%$, versus $17.68 \%$ in the emerging markets. This emerging market premium is reflective of an annual standard deviation of $33.63 \%$ versus only $18.41 \%$ in developed markets. Kurtosis is on average higher in emerging markets, indicating more tail risk. But skewness is slightly positive in emerging markets and slightly negative in mature markets, suggesting that emerging markets are not more risky from this perspective.

The first-order autocorrelations are small for most countries. The Ljung-Box (LB) test that the first 20 weekly autocorrelations are zero is not rejected in most developed markets but it is rejected in most emerging markets. We use an autoregressive model of order two, $\mathrm{AR}(2)$, for each market to pick up this return dependence. The Ljung-Box test that the first 20 autocorrelations in absolute returns are zero is strongly rejected for all 29 markets. We employ a GARCH model for each market to pick up this second-moment dependence. As discussed in Section 2.2, we use the 
NGARCH model of Engle and Ng (1993) in equation (2.2) and skewed $t$ innovations to account for univariate asymmetries.

Table 2 reports the results from the estimation of the $\operatorname{AR}(2)-\mathrm{NGARCH}(1,1)$ models with skewed $t$ innovations on each market for the 1989-2008 data set. The results are fairly standard. The volatility updating parameter, $\alpha$, is around 0.1 , and the autoregressive variance parameter, $\beta$, is around 0.8. The parameter $\gamma$ governs the volatility asymmetry and is also known as the leverage effect. It is commonly found to be large and positive in developed markets and we find that here as well for most countries. Interestingly, the average leverage effect is much closer to zero in the emerging markets and it is negative in quite a few cases. The model-implied variance persistence is high for all countries, as is commonly found in the literature.

The Ljung-Box (LB) tests on the model residuals show that the $\mathrm{AR}(2)$ models are able to pick up the weak evidence of return predictability found in Table 1. Impressively, the GARCH models are also able to pick up the strong persistence in absolute returns found in Table 1. Note also that the skewed $t$ GARCH model picks up much of the excess kurtosis found in Table 1.

We conclude from Tables 1 and 2 that the skewed $t \operatorname{AR}(2)-\mathrm{NGARCH}(1,1)$ models are successful in delivering the white-noise residuals that are required to obtain unbiased estimates of the dynamic copula correlations.

\subsection{Copula Correlation Patterns Over Time}

Table 3 reports the parameter estimates and composite log likelihood values for the DAC model. We report results for the three data sets introduced above. For each set of countries we estimate two versions of each model: one version allowing for copula correlation dynamics, and another where the correlation dynamics are completely shut down, and thus $\alpha_{\Gamma}=\beta_{\Gamma}=0$. A conventional likelihood ratio test would suggest that the last model is rejected for all sets of countries, but unfortunately the standard chi-squared asymptotics are not available for composite likelihoods.

The dependence persistence $\left(\alpha_{\Gamma}+\beta_{\Gamma}\right)$ is close to one in all models, implying very slow meanreversion in copula correlations, despite the presence of a trend.

Figure 1 presents time series of averages of the dynamic copula correlations across countries for several samples. The left panels in Figure 1 present results for twenty-nine developed and emerging markets for the sample period January 20, 1989 to July 25, 2008. We refer to this sample as the 1989-2008 sample. As explained in Section 3.1, sixteen of these markets are developed and thirteen are emerging markets.

The right panels in Figure 1 present results for thirty-three developed and emerging markets for the sample period July 21, 1995 to June 12, 2009. This sample contains the same sixteen 
developed markets, and seventeen emerging markets. There is considerable overlap between this sample of emerging markets and the one used in the left panels of Figure 1. Section 3.1 discusses the differences. We refer to this sample as the 1995-2009 sample.

The top panel in Figure 2 contains results for the group of sixteen developed markets between January 26, 1973 and June 12, 2009. We refer to this sample as the 1973-2009 sample. Figure 2 also shows results for the 1989-2008 and the 1995-2009 data for comparison.

These figures contain some of the main messages of our paper. The dynamic copula correlations in Figure 1 and 2 fluctuate considerably from year to year, but have been on an upward trend since the beginning of the sample. Figure 2 shows that for the sixteen developed markets, the average copula correlation increased from approximately 0.2 in the mid-1970s to around 0.8 in 2009. Figure 1 indicates that over the 1989-2009 period, the copula correlations between emerging markets are lower than those between developed markets, but that they have also been trending upward, from approximately 0.1-0.2 in the early nineties to over 0.5 in 2009. Figure 1 also indicates that the model-implied trend, indicated by the dashed line, is roughly linear for the emerging markets, while the increase in copula correlations has somewhat slowed in recent years for the developed markets.

When estimated on all markets, recall that our DAC model has two different time trend parameters; one for developed markets and one for emerging markets. The last row in Figure 1 presents the resulting time trend for cross-correlation between developed and emerging markets, which depends on these two parameters. While the first three rows of graphs indicate that correlation have increased within DMs, within EMs and on average across all markets, the bottom graphs confirm that average correlation between DMs and EMs have also increased.

Table 3 also reports the standard errors for all parameter estimates using the technique in Engle, Shephard and Sheppard (2008). ${ }^{10}$ The total increase in long run average correlation depends on the time trend parameters, $\delta_{D M}$ and $\delta_{E M}$, as well as on the weighting parameter $\varphi_{\Gamma}$. We report the increase in copula correlation for DMs over the sample that is due to the time trend component using (2.4) and (2.5) as follows

$$
\left\{\varphi_{\Gamma} \Upsilon_{D M, t}\right\}_{t=T}-\left\{\varphi_{\Gamma} \Upsilon_{D M, t}\right\}_{t=0}=\varphi_{\Gamma} \frac{\delta_{D M}^{2} T^{2}}{1+\delta_{D M}^{2} T^{2}}=\varphi_{\Gamma} \Upsilon_{D M, T}
$$

and similarly for EMs. The increases in long-run copula correlation ranges from 0.25 to 0.39 for DMs and 0.29 to 0.38 for EMs and are all positive and most often significant as seen from Table

${ }^{10}$ Asymptotic standard errors are computed using each bivariate element of the composite log-likelihood in equation (2.7). The standard errors are a function of the gradient and the Hessian of the copula parameters, the gradient of the moment estimator in equation (2.6), the gradient of the AR-NGARCH models, and their cross-derivatives. Please see Engle, Shephard and Sheppard (2008) for further detail. 
3. ${ }^{11}$ To illustrate further the importance of the evolution of the general level of dependence, we also report in Figure 1 and 2 the average constant copula correlation estimated on each sample along with bootstrapped $90 \%$ confidence intervals (dashed line and gray area). ${ }^{12}$ In all cases, both the dynamic and the long-run copula correlations are significantly lower than the constant copula correlation at the start of the sample, and higher at the end.

Because the model allows for dynamic copula correlations with a long-run trend, one may wonder whether the choice of sample period strongly affects inference on dependence estimates at a particular point in time. Figure 2 addresses this issue by reporting estimates for the sixteen developed markets for three different sample periods. Whereas there are some differences, the copula correlation estimate at a particular point in time is remarkably robust to the sample period used, and the conclusion that copula correlations have been trending upward clearly does not depend on the sample period used. Comparing the left and right panels of Figure 1, it can be seen that a similar conclusion obtains for emerging markets, even though this comparison is more tenuous, as the sample composition and the return data used for the emerging markets are somewhat different across panels.

\subsection{Cross-Sectional Differences in Copula Correlations}

The average copula correlations indicate that dependence has increased on average over our sample. The next question is how much cross-sectional heterogeneity there is in the copula correlations, and if the increases in dependence are different across countries and regions. ${ }^{13}$ Reporting on all these time-varying pairwise copula correlation paths is not feasible, and we have to aggregate the correlation information in some way. Figures 3-5 provide an overview of the results. ${ }^{14}$

Figure 3 uses the 1989-2008 sample to report, for each of the twenty-nine countries in the sample, the average of its copula correlations with all other countries using light grey lines. Figure 3.A contains the 16 developed markets and Figure 3.B contains the 13 emerging markets. While these paths are averages, they give a good indication of the differences between individual countries, and

\footnotetext{
${ }^{11}$ We compute asymptotic standard errors of the correlation trend increase using the delta method.

${ }^{12}$ In the bootstrap we generate 10,000 samples by randomly drawing with replacement from $\eta_{t}$ defined in equation (2.3). For each bootstrap sample, we compute the average pairwise copula correlation. Using the 10,000 average copula correlations, we then form a 90\% confidence interval around the constant copula correlation. Alternatively, we can construct confidence intervals around the time-varying copula correlations, but this is computationally much more expensive.

${ }^{13}$ A related literature explores the relationship between industrial structure and the dynamics of equity market returns and cross-country correlations. See for instance Roll (1992), Heston and Rouwenhorst (1994), Griffin and Karolyi (1998), and Dumas, Harvey and Ruiz (2003).

${ }^{14}$ Throughout the paper, we report equal-weighted averages of the pairwise copula correlations. Value-weighted correlations (not reported here) also display an increasing pattern over our samples.
} 
they are also informative of the differences between developed and emerging markets. In order to further study these differences, each figure also gives the average of the market's copula correlations with all (other) developed markets using black lines and all (other) emerging markets using dark grey lines. Figures 3.A and 3.B yield some very interesting conclusions. First, the copula correlation paths display an upward trend for all 29 countries, except perhaps Jordan. Second, for developed markets the average copula correlation with other developed markets is higher than the average copula correlation with emerging markets at virtually each point in time for virtually all markets. Third, for emerging markets the copula correlation with developed markets is generally higher than the copula correlation with other emerging markets. However, the difference between the two copula correlation paths is much smaller than in the case of developed markets, and in several cases the average paths are very similar. Note that in Figure 3.A the trend patterns for European countries are also not very different from those for other DMs. Notice that, even if their level is still somewhat lower, the correlations for Japan and the US have increased just as for the European countries during the last decade. Inspection of the pairwise DAC paths, which are not reported because of space constraints, reveals that the trend patterns are remarkably consistent for almost all pairs of countries, and there is no meaningful difference between European countries and other DMs.

Figure 3 reports the averages of the copula correlations between each market and all other markets. It could be argued that instead the correlation between each market and the average return of the other markets ought to be considered. We have computed these correlations as well, but we do not show them in order to save space. While the correlation with the average return is nearly always higher than the average correlation from Figure 3, the conclusion that the correlations are trending upwards is not affected.

We can use the correlation paths from the DAC model to assess regional patterns in correlation dynamics. Figure 4 does exactly this. We divide the $16 \mathrm{DMs}$ into two regions (EU and non-EU) and we divide the 13 EMs into another two EM regions: Latin America and Emerging Eurasia. ${ }^{15}$ We report in Figure 4 the average copula correlation within and across the four regions, based on the model's country-specific correlation paths. Strikingly, Figure 4 shows that the increasing dependence patterns are evident within each of the four regions and also across all the six possible pairs of regions. The highest levels of copula correlation are found in the upper-left panel which shows the intra-EU copula correlations. The lowest levels are found in the bottom-right panel which shows the intra Emerging Eurasia copula correlations. Emerging Eurasia in the right-most column

\footnotetext{
${ }^{15}$ The European Union (EU) includes Austria, Belgium, Denmark, France, Germany, Ireland, Italy, Netherlands, and the UK. Developed Non-EU includes Australia, Canada, Hong Kong, Japan, Singapore, Switzerland, and the US. Latin America includes Argentina, Brazil, Chile, Colombia, and Mexico. Emerging Eurasia includes India, Jordan, Korea, Malaysia, Philippines, Taiwan, Thailand, and Turkey.
} 
generally has the lowest interregional copula correlations.

Figures 3 and 4 do not tell the entire story, because we have to resort to reporting copula correlation averages due to space constraints. Figure 5 provides additional perspective by providing copula correlation dispersions for the developed markets, emerging markets, and all markets respectively. In particular, at each point in time, the shaded areas in Figure 5 show the range between the 10th and 90th percentile based on all pairwise copula correlations between groups of countries. The top panel considers the sixteen developed countries. The middle panel in Figure 5 reports the same statistics for the emerging markets for the 1989-2008 sample and the bottom panel shows all 29 markets together. While the increasing level of dependence is evident, the range seems to have narrowed for developed markets, widened a bit for emerging markets, and the range width seems to have stayed roughly constant for all markets taken together.

\section{Conditional Diversification Benefits}

If the level of dependence is changing over time, then the benefits of portfolio diversification are likely changing as well. We therefore need to develop a dynamic measure of diversification benefits that takes into account higher order moments and nonlinear dependence. Motivated by the analysis in Basak and Shapiro (2001), we develop a dynamic measure based on expected shortfall. We first discuss this measure in detail. We then provide more intuition by considering this measure under the special case of multivariate normality, and subsequently we report our empirical estimates.

\subsection{A Conditional Measure of Diversification Benefits}

Our approach is based on the expected shortfall measure defined as

$$
E S_{t}^{q}\left(R_{i, t}\right)=-E\left[R_{i, t} \mid R_{i, t} \leq F_{i, t}^{-1}(q)\right] .
$$

where $F_{i, t}^{-1}(q)$ is the inverse cumulative distribution function of asset $i$ at time $t$, and $q$ is a probability commonly set to $5 \%$ or $1 \%$. Expected shortfall is a coherent risk measure ${ }^{16}$ and so the upper bound on the portfolio $E S$ is the weighted average of the assets' individual expected shortfalls

$$
\overline{E S}_{t}^{q} \equiv \sum_{i=1}^{N} w_{i, t} E S_{t}^{q}\left(R_{i, t}\right)
$$

\footnotetext{
${ }^{16}$ See Artzner, Delbaen, Eber and Heath (1999).
} 
where $w_{i, t}$ is the portfolio weight on asset $i$ at time $t$. This corresponds to the case of no diversification benefits.

The lower bound on expected shortfall is

$$
\underline{E S}_{t}^{q} \equiv-F_{P, t}^{-1}(q)
$$

This corresponds to the extreme case where the portfolio never loses more than its qth quantile.

Using these two extreme cases, we define the conditional diversification benefit (CDB) measure by

$$
C D B_{t}\left(w_{t}, q\right) \equiv \frac{\overline{E S}_{t}^{q}-E S_{t}^{q}\left(w_{t}\right)}{\overline{E S}_{t}^{q}-\underline{E S}_{t}^{q}} .
$$

where $E S_{t}^{q}\left(w_{t}\right)$ denotes the expected shortfall of the portfolio at hand. This measure is designed to take values on the $[0,1]$ interval, and is increasing in the level of diversification benefit. Note also that it does not depend on the level of expected returns.

Below we report on the case where $q$ is $5 \%$ and we choose $w_{t}$ to maximize $C D B_{t}\left(w_{t}, q\right)$ subject to the weights being non-negative and summing to one. ${ }^{17}$

In order to assess how much of the conditional diversification benefit derives from active asset allocation, we also construct a $C D B_{t}^{E W}$ measure for an equal-weighted portfolio. By definition $C D B_{t}^{E W}$ will be less than or equal to its optimal counterpart $C D B_{t}$ at any point in time. The difference between these two measures indicates if equal-weighted portfolios are close to optimal, and measures to what extent changing volatilities and dependence can be exploited via dynamic asset allocation.

\subsection{The Special Case of Normality}

Our $C D B_{t}$ measure is designed to capture nonnormalities and simplifies greatly when returns are normally distributed. Under normality, we have

$$
E S^{q}=-\mu+\sigma \frac{\phi\left(\Phi^{-1}(q)\right)}{q}
$$

where $\mu$ and $\sigma$ are the mean and the standard deviation of returns, and $\phi$ and $\Phi$ are the standard normal density and cumulative distribution functions, respectively. Substituting in equation (4.1), we obtain

$$
C D B_{t}\left(w_{t}, q\right)=\frac{w_{t}^{\top} \sigma_{t}-\sigma_{P, t}}{w_{t}^{\top} \sigma_{t}+\sigma_{P, t} \frac{\Phi^{-1}(q) q}{\phi\left(\Phi^{-1}(q)\right)}}
$$

\footnotetext{
${ }^{17}$ The results for different values of $q$ are qualitatively similar and available from the authors upon request.
} 
where $\sigma_{t}$ denotes the vector of individual asset volatilities at time $t$ and $\sigma_{P, t}$ is portfolio volatility. Note that in the special case of $q=50 \%$, the measure reduces to

$$
\operatorname{VolCD} B_{t}\left(w_{t}\right) \equiv C D B_{t}\left(w_{t}, 0.5\right)=\frac{w_{t}^{\top} \sigma_{t}-\sigma_{P, t}}{w_{t}^{\top} \sigma_{t}}=1-\frac{\sqrt{w_{t}^{\top} \Sigma_{t} w_{t}}}{w_{t}^{\top} \sigma_{t}},
$$

where $\Sigma_{t}$ denotes the variance-covariance matrix of returns. The denominator $w_{t}^{\top} \sigma_{t}$ represents portfolio volatility in the extreme case of no diversification benefits, and $\operatorname{Vol} C D B_{t}$ measures conditional diversification benefits as the degree of portfolio volatility reduction from this upper bound. We report $\operatorname{Vol} C D B$ estimates in our empirical analysis below along with the more general $C D B$ estimates.

\subsection{Diversification Benefits}

Figure 6 plots the conditional diversification benefit measures developed in equations (4.1) and (4.3) for developed, emerging, and all markets, evaluated using our estimates of the DAC model. The left-hand panels present results for the general measure in (4.1), and the right-hand panels present results for the special case in (4.2) where only the volatilities and linear correlations from the model are used.

The top-left panel shows a clearly decreasing trend in diversification benefits in DMs for both the optimal (black) and equal-weighted (grey) allocations: Dependence has been rising rapidly and the benefits of diversification have been decreasing during the last ten years. Diversification benefits have also decreased in emerging markets (middle left panel) but the level of benefit is still higher than in developed markets. When combining the developed and emerging markets (bottom left

panel), the diversification benefits are declining as well but the level is again much higher than when considering developed markets alone. Emerging markets thus offer substantial diversification benefits to investors.

In the case of the volatility-based measure in the right-side panels, similar conclusions obtain, but there are some interesting differences. Compared to the general $C D B$ case, the declining $\operatorname{VolCDB}$ trend seems more pronounced for emerging markets. We therefore conclude that the diversification benefits offered by emerging markets are partly due to the diversification of large market downturns.

For both the general $C D B$ and the $\operatorname{Vol} C D B$ measures, the differences between the optimal and the equally-weighted portfolio are nonzero, but not very large. The differences are somewhat larger for the volatility based measure in the right panels and largest when considering EMs and DMs jointly in the bottom-right panel. Overall, the relatively modest differences between optimal and equal-weighted diversification benefits suggest that the " $1 / N$ " style portfolios recently advocated 
in a normal setting may work relatively well in our nonnormal context as well. ${ }^{18}$

\section{$5 \quad$ Further Economic Implications}

The declining diversification benefits constitute an important implication of the rising dependence across international equity markets. We now explore some further economic implications of our empirical estimates. We document the models' implications for tail dependence, and investigate if the models can match well-known stylized facts regarding threshold correlations. We also derive the implications of our estimates for copula correlations over longer horizons. Finally, we use regression analysis to assess how equity market correlations are related to market openness as well as to financial and macro variables more generally.

\subsection{Tail Dependence}

Tables 4 and 5 present additional estimation results for two nested copula models, the dynamic symmetric $t$ copula (DSC) and the dynamic normal copula (DNC). This provides an alternative way to assess the importance of asymmetries and nonnormalities in the copula, as opposed to the statistical significance of the estimates of $\nu$ and $\lambda$ in Table 3. While we cannot use standard asymptotics to compare the composite likelihoods, it is clear that the DAC likelihoods in Table 3 are much larger than the DSC likelihoods in Table 4 and the DNC likelihoods in Table 5. Note that the copula correlation persistence is-as was the case in Table 3-very close to one in all models. To further illustrate the differences between the DAC model and the two nested cases, we now discuss model-implied tail dependence coefficients, while the next section discusses threshold correlations.

The DAC model generalizes the DNC model by allowing for non-zero dependence in the tails, and it generalizes the DSC model by allowing for different dependence in the lower and upper tails. One way to measure the lower tail dependence is via the probability limit

$$
\tau_{i, j, t}^{L}=\lim _{\zeta \rightarrow 0} \operatorname{Pr}\left[\eta_{i, t} \leq \zeta \mid \eta_{j, t} \leq \zeta\right]=\lim _{\zeta \rightarrow 0} \frac{C_{t}(\zeta, \zeta)}{\zeta}
$$

where $\zeta$ is the tail probability. The upper tail dependence at time $t$ can similarly be defined by

$$
\tau_{i, j, t}^{U}=\lim _{\zeta \rightarrow 1} \operatorname{Pr}\left[\eta_{i, t} \geq \zeta \mid \eta_{j, t} \geq \zeta\right]=\lim _{\zeta \rightarrow 1} \frac{1-2 \zeta+C_{t}(\zeta, \zeta)}{1-\zeta}
$$

\footnotetext{
${ }^{18}$ In the multivariate normal case, DeMiguel, Garlappi and Uppal (2009) and Tu and Zhou (2011) analyze the relative performance of equal-weighted versus optimally-weighted portfolios in an unconditional setting.
} 
The normal copula has the empirically questionable property that its tail dependence is zero. The tail dependence is positive in DAC and DSC models we develop, and the tail dependence measure depends on the degree of freedom, $\nu$, the copula correlation, $\Psi_{i, j}$, and the skewness parameter, $\lambda .^{19}$

In the DSC model the lower and upper tail dependence is identical, that is $\tau_{i, j, t}^{L}=\tau_{i, j, t}^{U}$. Based on the work by Longin and Solnik (2001) and Ang and Bekaert (2002), we suspect that such symmetry does not characterize international equity index returns, and we therefore investigate the difference between upper and lower tail dependence using the DAC model. ${ }^{20}$

Figure 7 plots the dynamic measure of tail-dependence in equations (5.1) and (5.2) for the DAC model. We report the average of the bivariate tail dependence across all pairs of countries. ${ }^{21}$ In each graph, the dark line depicts the evolution of the lower tail dependence, while the gray line is for the upper tail dependence. ${ }^{22}$ Figure 7 shows quite dramatic differences across markets. The tail dependence in developed markets has risen markedly during the last twenty years. Remarkably, while the emerging market tail dependence measures in the middle panel of Figure 7 have also increased, they remain very low compared to developed markets. When considering all markets in the bottom panel of Figure 7, we find that while the tail dependence is rising, it is still much lower than for the developed markets alone. From this perspective, the diversification benefits from adding emerging markets to a portfolio appear to be large compared to those offered by developed markets alone, even if these benefits have become smaller over time. In all cases, lower tail dependence is higher than upper tail dependence, suggesting substantial negative skewness in the multivariate distribution of international equity returns.

The relatively modest tail dependence found for EMs in Figure 7 helps explain the diversification benefits offered by EMs in the left panels of Figure 6 where the general CDB measure is used.

\subsection{Threshold Correlations}

For each pair of countries we compute threshold correlations from return shocks, as follows

$$
\widetilde{\rho}_{\pi}\left(z_{i}, z_{j}\right)= \begin{cases}\operatorname{Corr}\left(z_{i}, z_{j} \mid z_{i} \leq \pi, z_{j} \leq \pi\right) & \text { if } \pi \leq 0 \\ \operatorname{Corr}\left(z_{i}, z_{j} \mid z_{i}>\pi, z_{j}>\pi\right) & \text { if } \pi>0\end{cases}
$$

\footnotetext{
${ }^{19}$ See Patton (2006) for an application of the tail dependence measure to exchange rates.

${ }^{20}$ On tail dependence, see also Poon, Rockinger, and Tawn (2004). On the related topic of contagion, see for example Forbes and Rigobon (2002), Bekaert, Harvey, and Ng (2005), and Bae, Karolyi, and Stulz (2003).

${ }^{21}$ The tail dependence concept introduced above is inherently bivariate and not easily generalized to higher dimensions. In order to convey the empirical evolution of tail dependence for many countries, we report the average of the bivariate tail dependence across all pairs of countries.

${ }^{22}$ To the best of our knowledge a closed form solution is not available for the tail dependence measure in the case of the skewed $t$ copula. We therefore approximate it by numerical integration using $\zeta=0.001$.
} 
where $z_{i}$ are shocks for country $i$. We also compute similar threshold correlations based on standardized returns. Figure 8 plots the pairwise empirical threshold correlations averaged across countries. The left panels report the threshold correlations corresponding to returns, which are standardized by their unconditional mean and standard deviations, and the right panels report the threshold correlations computed on shocks, which are returns standardized by the AR-NGARCH model with skewed $t$ innovations discussed in Section 2.2. The correlations are computed for a grid of thresholds $\pi$ (denoted in standard deviations), provided that at least twenty observations are available. The dashed line reports threshold correlations implied by a normal distribution using the average linear correlation estimated from the data. As is well-known, asymmetric threshold correlations cannot be captured using a multivariate normal distribution: threshold correlations in the normal distribution are symmetric, and also decrease rather quickly in the tails.

When considering monthly returns in the US versus other DMs, Longin and Solnik (2001) found that the downside threshold correlations were much larger than their upside counterparts. The solid black line in the top left panel of Figure 8 confirms the findings of Longin and Solnik (2001): When computing the average of all possible pairwise threshold correlations for weekly returns in sixteen DMs we find that the downside threshold correlations indeed are much larger than upside threshold correlations regardless of how returns are standardized.

The bottom left panel of Figure 8 shows the average threshold correlations for EMs, which has not yet been documented in the literature. Threshold correlations are also asymmetric for EMs. As in the case of DMs, the downside threshold correlations are larger than upside threshold correlations. When comparing the top and bottom panels of Figure 8 we see that the downside threshold correlations are higher for developed markets than for emerging markets, which is consistent with our earlier findings.

The comparison of the left-side panels based on returns and the right-side panels based on shocks is very instructive to illustrate the advantages of copula modeling, and of the DAC model in particular. The asymmetries and nonnormalities built into the model for the marginals do generate multivariate asymmetries and nonnormalities. If these asymmetries and nonnormalities are sufficient to capture the stylized facts in the data, we should not observe asymmetries in the right-side panels. This is clearly not the case. In fact, the right-side panels are very similar to the left-side panels, indicating the limited role that the modeling of the marginals can play in capturing multivariate asymmetries, and emphasizing the need for asymmetric copula models.

To see how well our models fare, we follow the empirical setup in Ang and Bekaert (2002) and compare the pattern in empirical threshold correlations with threshold correlations from simulated data generated using the models. Figure 9 presents the threshold correlations for NGARCH shocks implied by the DAC and DSC models. Note that the DSC model (marked by ' + ') is successful 
in producing higher threshold correlations than the Gaussian distribution, but by design these correlations are also symmetric. The DAC model (marked by ' $\bigcirc$ ') produces an asymmetric pattern in threshold correlations, with substantially higher downside threshold correlations.

The DAC model does not capture the threshold correlation patterns perfectly. This is not surprising, because its parameters optimize the composite likelihood and not the distance between model based threshold correlations and their empirical counterpart. Moreover, the averaging of empirical threshold correlations and model parameters may induce biases in the empirical comparison. To demonstrate the model's potential, we simulate data using a bivariate DAC model with the average values for all parameter estimates, except for $\lambda$, which we set equal to minus one to roughly match the threshold asymmetry in the data. The threshold correlation (marked by ' $\times$ ') shows that if the objective is to match the average level of threshold correlation, the DAC model is able to do so.

\subsection{Implied Copula Correlations for Longer Horizons}

We have used weekly returns in order to be able to capture volatility and dependence dynamics while avoiding non-synchronicity and other problems with daily data. However, not all investors have a weekly horizon. Hence, we now consider the implications of our findings for longer horizons. The left panels in Figure 10 present monthly copula correlations averaged across countries, while the right panels report annual copula correlations. Each month (year), we simulate returns from the AR-NGARCH and DAC models over the next 4 (52) weeks, compound them, and compute the copula correlations of the resulting returns. Not surprisingly, the long horizon copula correlations are smoother than the shorter horizon correlations. Further, the dependence is clearly increasing over time for DMs as well as EMs and confirms our earlier finding that the dependence between EMs is lower than between DMs.

\subsection{Correlation Regressions}

In this section we ask how the copula correlations plotted in Figure 3 are related to volatility, market openness, and financial and macro variables more generally. We run country level panel regressions on the copula correlations from the DAC model. Table 6 reports the results in four panels. The regressand in Panel A is the natural logarithm of the average developed market copula correlation with other developed markets. In Panel B it is the natural logarithm of the average developed market copula correlation with all emerging markets. Panels $\mathrm{C}$ and $\mathrm{D}$ report on the natural logarithm of the average emerging market copula correlations with all developed markets and all other emerging markets respectively. Due to macro data availability, the data frequency is 
quarterly, and our sample is restricted between the third quarter of 1995 and the second quarter of 2008. We include a quadratic trend in all specifications and assess if the explanatory variables are able to render the correlation trend insignificant. The specifications labeled (i) in Table 6 contain only the trend which is significant throughout Panels A-D.

Factor models typically imply a positive relationship between correlation and volatility. Because we obtain our results without the aid of a factor model, it is worth investigating if this positive relationship is confirmed. Specification (ii) reports the results in each of the panels. We regress correlation on the average log GARCH volatility across EM or DM countries and find a positive relationship throughout Panels A-D. Comparing the results of specification (ii) with those of specification (i) we see that including volatility in the regression does not affect the trend coefficient estimates nor the statistical significance of the estimated coefficients much. ${ }^{23}$ We conclude that the documented trend in correlation cannot be explained by volatility movements.

We now investigate the relationship between financial development and dependence in emerging markets. In order to measure financial development, we rely on Bekaert (1995) and Edison and Warnock (2003), who use a direct measure of de jure market openness. Their measure is defined as the ratio of the market capitalizations of the investable (IFCI) and global (IFCG) indexes from $\mathrm{S} \& \mathrm{P} / \mathrm{IFC}$, and we denote it by " $M C R$ ". When the $M C R$ measure is one, the market capitalization of the investable index is equal to that of the market-wide index. We report the regression results in specification (iii) of Panels $\mathrm{C}$ and $\mathrm{D}$. The relationship between the openness indicator $M C R$ and dependence is statistically significant at the $5 \%$ level in both cases, and the estimate is positive as expected. However, the estimates of the time trend coefficients are little affected and still statistically significant.

Finally we regress copula correlations on the following financial variables: the volatility risk premium defined as the VIX less a moving 22-day standard deviation on daily index returns; the 3-month U.S. T-bill; the U.S. term spread (10-year less 1-year); the U.S. credit spread; and the average of the real interest rate in the G7 economies. We also include $M C A P$ defined as the market value divided by the preceding year's GDP. Finally, we include turnover, which is measured as trading value divided by market capitalization, and which serves as a liquidity proxy. In addition we use the following macro variables: the world real GDP growth rate, and the ratio of the sum of exports and imports of goods and services to the gross domestic product denoted by "Trade" ${ }^{24}$

We have investigated all permutations of the financial and macroeconomic variables, but for

\footnotetext{
${ }^{23}$ Following Petersen (2009), we compute White standard errors adjusted for within clusters (country and quarter) correlation.

${ }^{24}$ Our variable selection is motivated by the work of Schwert (1989), Engle, Ghysels, and Sohn (2008), Engle and Rangel (2008), Boyer, Mitton, and Vorkink (2010), Ghysels, Plazzi, and Valkanov (2011), Bekaert, Harvey, Lundblad, and Siegel (2012), Carrieri, Chaieb, and Errunza (2011), and Baele, Bekaert, and Inghelbrecht (2010).
} 
brevity we report the most general specification including all variables. The results are in specification (iii) for Panels $\mathrm{A}$ and $\mathrm{B}$, and in specification (iv) for Panels $\mathrm{C}$ and D. We find that it is possible to identify financial variables that affect international return dependence, even with these small samples. The volatility risk premium coefficient is significantly positive in all cases. The U.S. 3-month T-bill rate coefficient is significantly negative in all cases. The point estimate on the U.S. term spread is negative in all cases, and it is statistically significant in three of four cases. The credit spread is significant in Panel D only. The G7 real rate positively impacts dependence but it is only significant in two of four cases. $M C A P$ is not significant and turnover is significantly positive in Panel A and B only. In terms of the macro variables, the World GDP growth is positive in all cases and significant in three out of four. The trade variable is not significant.

Finally, note that in Table 6, although the various variables we have considered are often significant, the time trend coefficients remain highly significant. We therefore conclude that the long-run dynamics in dependence cannot be explained by standard macroeconomic and financial variables. Clearly, these results raise important questions as to which factors drive cross-country dependence.

\section{Summary and Conclusion}

We characterize time-varying dependence using long samples of weekly returns for a large number of countries. We propose a new dynamic asymmetric copula model that can capture dynamic dependence while accommodating multivariate nonnormality, asymmetries, and trends in dependence. We overcome econometric complications arising from the dimensionality problem using the composite likelihood procedure.

Our results are extremely robust and suggest that dependence has significantly trended upward for both DMs and EMs. Copula correlations between DMs have exceeded copula correlations between EMs throughout the 1989-2009 period. Moreover, for developed markets, the average dependence with other developed markets is higher than the average dependence with emerging markets. For emerging markets, the dependence with developed markets is generally somewhat higher than the dependence with the other emerging markets, but the differences are small. While the range of copula correlations for DMs has narrowed around the increasing trend in correlation levels, this is not the case for EMs.

We find substantial evidence of asymmetric tail dependence with lower tail dependence being larger than upper tail dependence. Moreover, tail dependence as computed from the DAC model is large and increasing through time for DMs, but remains low for EMs. We propose a new measure of diversification benefits that takes into account tail dependence, and find that whereas diversification benefits have largely disappeared for DMs, EMs still offer substantial diversification benefits. From 
this perspective, our results suggest that although diversification benefits have lessened in the case of DMs, the case for EMs remains strong. This is due to the fact that while equity market crises in EMs are frequent, many of them are country-specific.

These results have very important implications for portfolio management, and it may prove interesting to further explore them in future work. It may also prove useful to investigate the robustness of our findings to allowing for multiple regimes, or to the inclusion of multiple stochastic components, as for example in the model of Colacito, Engle, and Ghysels (2011). 


\section{References}

[1] Aielli, G. P., 2009, Dynamic Conditional Correlations: On Properties and Estimation, Working paper: Department of Statistics, University of Florence.

[2] Ang, A., and G. Bekaert, 2002, International Asset Allocation with Regime Shifts, Review of Financial Studies 15, 1137-1187.

[3] Ang, A. and J. Chen, 2002, Asymmetric Correlations of Equity Portfolios, Journal of Financial Economics 63, 443-494.

[4] Artzner, P., Delbaen, F. Eber, J.-M. and D. Heath, 1999, Coherent Measures of Risk, Mathematical Finance 9, 203-228.

[5] Bae, K.-H., Karolyi, G.A., and R. Stulz, 2003, A New Approach to Measuring Financial Contagion, Review of Financial Studies 16, 717-763.

[6] Baele, L., and K. Inghelbrecht, 2009, Time-Varying Integration and International Diversification Strategies, Journal of Empirical Finance 16, 368-387.

[7] Baele, L., Bekaert, G., and K. Inghelbrecht, 2010, The Determinants of Stock and Bond Return Comovements, Review of Financial Studies 23, 2374-2428.

[8] Basak, S. and A. Shapiro, 2001, Value-at-Risk-Based Risk Management: Optimal Policies and Asset Prices, Review of Financial Studies 14, 371-405.

[9] Bekaert, G., 1995, Market Integration and Investment Barriers in Emerging Equity Markets, The World Bank Economic Review 1, 75-107.

[10] Bekaert, G., and C. Harvey, 2000, Foreign Speculators and Emerging Equity Markets, Journal of Finance 55, 565-613.

[11] Bekaert, G., Harvey, C., Lundblad, C. and S. Siegel, 2011, What Segments Equity Markets? Review of Financial Studies 24, 3841-3890.

[12] Bekaert, G., Harvey, C., and A. Ng, 2005, Market Integration and Contagion, Journal of Business 78, 39-69.

[13] Bekaert, G., Hodrick, R., and X. Zhang, 2009, International Stock Return Comovements, Journal of Finance 64, 2591-2626. 
[14] Bekaert, G., and G. Wu , 2000, Asymmetric Volatility and Risk in Equity Markets, Review of Financial Studies 13, 1-42.

[15] Black, F., 1976, Studies of Stock Price Volatility Changes, In: Proceedings of the 1976 Meetings of the Business and Economic Statistics Section, American Statistical Association, 177-181.

[16] Boyer, B., Mitton, T., and K. Vorkink, 2010, Expected Idiosyncratic Skewness, Review of Financial Studies 23, 169-202.

[17] Brooks, R., and M. Del Negro, 2003, The Rise in Comovement Across National Stock Markets: Market Integration or IT Bubble? Journal of Empirical Finance 11, 659-680.

[18] Carrieri, F., Errunza, V., and K. Hogan, 2007, Characterizing World Market Integration Through Time, Journal of Financial and Quantitative Analysis 42, 915-940.

[19] Carrieri, F., Chaieb, I., and V. Errunza, 2011, Do Implicit Barriers Matter for Globalization?, Working Paper, McGill University.

[20] Chen, X., and Y. Fan, 2006, Estimation and Model Selection of Semiparametric Copula-Based Multivariate Dynamic Models under Copula Misspecification, Journal of Econometrics 135, 125-154.

[21] Colacito, R., Engle, R., and E. Ghysels, 2011, A Component Model for Dynamic Correlations, Journal of Econometrics 164, 45-59.

[22] Demarta, S., and A. J. McNeil, 2004, The $t$ Copula and Related Copulas, International Statistical Review 73, 111-129.

[23] DeMiguel, V., L. Garlappi and R. Uppal, 2009, Optimal versus Naive Diversification: How Inefficient Is the 1/N Portfolio Strategy? Review of Financial Studies 22, 1915-1953.

[24] DeSantis, G., and B. Gerard, 1997, International Asset Pricing and Portfolio Diversification with Time-Varying Risk, Journal of Finance 52, 1881-1912.

[25] Dumas, B., Harvey, C., and P. Ruiz, 2003, Are Correlations of Stock Returns Justified by Subsequent Changes in National Outputs? Journal of International Money and Finance 22, $777-811$.

[26] Edison, H. J. and F. E. Warnock, 2003. A Simple Measure of the Intensity of Capital Controls, Journal of Empirical Finance, 10, 81-103. 
[27] Eiling, E., and B. Gerard, 2007, Dispersion, Equity Return Correlations and Market Integration, Working Paper, University of Toronto.

[28] Engle, R., 2002, Dynamic Conditional Correlation: A Simple Class of Multivariate GARCH Models, Journal of Business and Economic Statistics 20, 339-350.

[29] Engle, R., Ghysels, E., and B. Sohn, 2008, On the Economic Sources of Stock Market Volatility, Working Paper, New York University.

[30] Engle, R., and V. Ng, 1993, Measuring and Testing the Impact of News on Volatility, Journal of Finance 48, 1749-1778.

[31] Engle, R., and J. Rangel, 2008, The Spline GARCH Model for Low Frequency Volatility and its Global Macroeconomic Causes, Review of Financial Studies 21, 1187-1222.

[32] Engle, R., Shephard, N., and K. Sheppard, 2008, Fitting Vast Dimensional Time-Varying Covariance Models, Working Paper, New York University.

[33] Erb, C., Harvey, C., and T. Viskanta, 1994, Forecasting International Equity Correlations, Financial Analysts Journal 4, 32-45.

[34] Errunza, V., 1977, Gains from Portfolio Diversification into Less Developed Countries' Securities, Journal of International Business Studies 55, 83-99.

[35] Errunza, V., Hogan, K., and M-W. Hung, 1999, Can the Gains from International Diversification be Achieved without Trading Abroad? Journal of Finance 54, 2075-2107.

[36] Forbes, K., and R. Rigobon, 2002, No Contagion, Only Interdependence: Measuring Stock Market Co-Movements, Journal of Finance 57, 2223-2261.

[37] Ghysels, E., A. Plazzi, and R. Valkanov, 2011, Conditional Skewness of Stock Market Returns in Developed and Emerging Markets and its Economic Fundamentals, Working Paper, University of North Carolina.

[38] Goetzmann, W., Li, L., and K. G. Rouwenhorst, 2005, Long-Term Global Market Correlations, Journal of Business 78, 1-38.

[39] Griffin, J., and A. G. Karolyi, 1998, Another Look at the Role of the Industrial Structure of Markets for International Diversification Strategies, Journal of Financial Economics 50, 351373. 
[40] Hansen, B., 1994, Autoregressive Conditional Density Estimation, International Economic Review $35,705-730$.

[41] Heston, S., and G. K. Rouwenhorst, 1994, Does Industrial Structure Explain the Benefits of International Diversification? Journal of Financial Economics 36, 3-27.

[42] Karolyi, G.A., and R. Stulz, 1996, Why do Markets Move Together? An Investigation of U.S.-Japan Stock Return Comovements, Journal of Finance 51, 951-986.

[43] King, M., Sentana, E., and S. Wadhwani, 1994, Volatility and Links Between National Stock Markets, Econometrica 62, 901-933.

[44] Kroner, K., and V. Ng, 1998, Modeling Asymmetric Comovement of Asset Returns, Review of Financial Studies 11, 817-844.

[45] Lewis, K.K., 2006, Is the International Diversification Potential Diminishing? Foreign Equity Inside and Outside the US, Working Paper, the Wharton School at the University of Pennsylvania.

[46] Longin, F., and B. Solnik, 2001, Extreme Correlation of International Equity Markets, Journal of Finance 56, 649-676.

[47] Marsaglia, G., and I. Olkin, 1984, Generating Correlation Matrices, SIAM Journal on Scientific and Statistical Computing 5, 470-475.

[48] McNeil, A., 1999, Extreme Value Theory for Risk Managers, Internal Modeling CAD II, Risk Books, 93-113.

[49] McNeil, A., and R. Frey, 2000, Estimation of Tail-Related Risk Measures for Heteroscedastic Financial Time Series: an Extreme Value Approach, Journal of Empirical Finance 7, 271-300.

[50] McNeil, A., Frey, R., and P. Embrechts, 2005, Quantitative Risk Management, Princeton: Princeton University Press.

[51] Patton, A., 2004, On the Out-of-Sample Importance of Skewness and Asymmetric Dependence for Asset Allocation, Journal of Financial Econometrics 2, 130-168.

[52] Patton, A., 2006, Modelling Asymmetric Exchange Rate Dependence, International Economic Review 47, 527-556. 
[53] Petersen, M. A., 2009, Estimating Standard Errors in Finance Panel Data Sets: Comparing Approaches, Review of Financial Studies 22, 435-480.

[54] Poon, S.-H., Rockinger, M., and J. Tawn, 2004, Extreme Value Dependence in Financial Markets: Diagnostics, Models, and Financial Implications, Review of Financial Studies 17, 581-610.

[55] Rangel, J., 2011, FX Comovements: Disentangling the Role of Market Factors, Carry-trades and Idiosyncratic Components, Working Paper, Banco de Mexico.

[56] Roll, R., 1992, Industrial Structure and the Comparative Behavior of International Stock Market Indices, Journal of Finance 47, 3-42.

[57] Schwert, G. W., 1989, Why Does Stock Market Volatility Change Over Time? Journal of Finance 44, 1115-1153.

[58] Sklar, A., 1959, Fonctions de Répartition à N Dimensions et Leurs Marges, Publications de l'Institut de Statistique de L'Université de Paris 8, 229-231.

[59] Solnik, B., 1974, The International Pricing of Risk: An Empirical Investigation of the World Capital Market Structure, Journal of Finance 29, 365-378.

[60] Solnik, B., and J. Roulet, 2000, Dispersion as Cross-Sectional Correlation, Financial Analysts Journal 56, 54-61.

[61] Tse, Y., and A. Tsui, 2002, A Multivariate GARCH Model with Time-Varying Correlations, Journal of Business and Economic Statistics 20, 351-362.

[62] Tu, J. and G. Zhou, 2011, Markowitz Meets Talmud: A Combination of Sophisticated and Naive Diversification Strategies, Journal of Financial Economics 99, 204-215. 


\section{Appendix}

\section{Appendix A. The Skewed $t$ Copula}

The skewed $t$ distribution discussed in Demarta and McNeil (2005) has the stochastic representation

$$
X=\sqrt{W} Z+\lambda W
$$

where $\lambda$ is the asymmetry parameter, $W$ is an inverse gamma variable $W \sim I G\left(\frac{\nu}{2}, \frac{\nu}{2}\right), Z$ is a normal variable $Z \sim N\left(0_{N}, \Psi\right)$, and $Z$ and $W$ are independent. The skewed $t$ distribution generalizes the $t$ distribution by adding a second term related to the same inverse gamma random variable which is scaled by a vector $\lambda$ of asymmetry parameters. Note that we constrain the copula to have the same asymmetry parameter across all assets, that is, $\lambda_{i}=\lambda_{j}$ for all $i, j$.

The probability density function of the skewed $t$ copula defined from the skewed $t$ distribution is given by

$$
\begin{aligned}
c(u ; \lambda, \nu, \Psi)= & \frac{2^{\frac{(\nu-2)(N-1)}{2}} K_{\frac{\nu+N}{2}}\left(\sqrt{\left(\nu+z^{* \top} \Psi^{-1} z^{*}\right) \lambda^{\top} \Psi^{-1} \lambda}\right) e^{z^{* \top} \Psi^{-1} \lambda}}{\Gamma\left(\frac{\nu}{2}\right)^{1-N}|\Psi|^{\frac{1}{2}}\left(\sqrt{\left(\nu+z^{* \top} \Psi^{-1} z^{*}\right) \lambda^{\top} \Psi^{-1} \lambda}\right)^{-\frac{\nu+N}{2}}\left(1+\frac{1}{\nu} z^{* \top} \Psi^{-1} z^{*}\right)^{\frac{\nu+N}{2}}} \\
& \times \prod_{j=1}^{N} \frac{\left(\sqrt{\left(\nu+\left(z_{j}^{*}\right)^{2}\right) \lambda_{j}^{2}}\right)^{-\frac{\nu+1}{2}}\left(1+\frac{1}{\nu}\left(z_{j}^{*}\right)^{2}\right)^{\frac{\nu+1}{2}}}{K_{\frac{\nu+1}{2}}\left(\sqrt{\left(\nu+\left(z_{j}^{*}\right)^{2}\right) \lambda_{j}^{2}}\right) e^{z_{j}^{*} \lambda_{j}}}
\end{aligned}
$$

where $K(\cdot)$ is the modified Bessel function of third kind, and where the copula shocks $z_{i}^{*}=t_{\lambda, \nu}^{-1}\left(u_{i}\right)$ are defined from the skewed univariate student $t$ density defined by

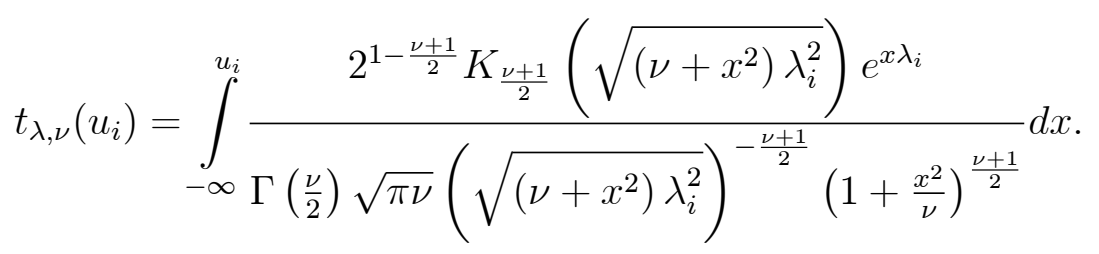

The skewed Student $t$ quantile function, $t_{\lambda, \nu}^{-1}\left(u_{i}\right)$, is not known in closed form but can be well approximated by simulating 100,000 replications of equation (6.1).

The moment of order $i$ of the $W$ variable is given by $m_{i}=\nu^{i} /\left(\prod_{j=1}^{i}(\nu-2 j)\right)$, and from the 
normal mixture structure of the distribution, we can derive the expected value

$$
E[X]=E(E[X \mid W])=E(W) \lambda=\frac{\nu}{\nu-2} \lambda
$$

and the variance-covariance matrix

$$
\begin{aligned}
\operatorname{Cov}(X) & =E(\operatorname{Var}(X \mid W))+\operatorname{Var}(E[X \mid W]) \\
& =\frac{\nu}{\nu-2} \Psi+\frac{2 \nu^{2} \lambda^{2}}{(\nu-2)^{2}(\nu-4)}
\end{aligned}
$$

Notice that the covariances are finite if $\nu>4$. These moments provide the required link between the multivariate skewed $t$ distribution and the copula correlation matrix $\Psi$.

We also provide results for the conventional symmetric $t$ distribution, which is nested when $\lambda$ tends to zero. The symmetric $N$-dimensional $t$ distribution has the stochastic representation

$$
X=\sqrt{W} Z
$$

where $W$ is an inverse gamma variable $W \sim I G\left(\frac{\nu}{2}, \frac{\nu}{2}\right), Z$ is a normal variable $Z \sim N\left(0_{N}, \Psi\right)$, and where $Z$ and $W$ are independent.

The probability density function of the $t$ copula defined from the $t$ distribution is given by

$$
c(u ; \nu, \Psi)=\frac{\Gamma\left(\frac{\nu+N}{2}\right)}{|\Psi|^{\frac{1}{2}} \Gamma\left(\frac{\nu}{2}\right)}\left(\frac{\Gamma\left(\frac{\nu}{2}\right)}{\Gamma\left(\frac{\nu+1}{2}\right)}\right)^{N} \frac{\left(1+\frac{1}{\nu} z^{* \top} \Psi^{-1} z^{*}\right)^{-\frac{\nu+N}{2}}}{\prod_{j=1}^{N}\left(1+\frac{z_{j}^{* 2}}{\nu}\right)^{-\frac{\nu+1}{2}}}
$$

where $z_{i}^{*}=t_{\nu}^{-1}\left(u_{i}\right)$ and $t_{\nu}\left(u_{i}\right)$ is the univariate Student's $t$ density function given by

$$
t_{\nu}\left(u_{i}\right)=\int_{-\infty}^{u_{i}} \frac{\Gamma\left(\frac{\nu+1}{2}\right)}{\sqrt{\pi \nu} \Gamma\left(\frac{\nu}{2}\right)}\left(1+\frac{x^{2}}{\nu}\right)^{-\frac{\nu+1}{2}} d x
$$

The Normal Copula is further nested as $\nu \rightarrow \infty$. The probability density function of the normal copula defined from the normal distribution is given by

$$
c(u ; \Psi)=\frac{1}{|\Psi|^{\frac{1}{2}}} e^{-\frac{1}{2} z^{* \top}\left(\Psi^{-1}-I_{N}\right) z^{*}}
$$


where $z_{i}^{*}=\Phi^{-1}\left(u_{i}\right)$ and $\Phi\left(u_{i}\right)$ is the univariate normal density function given by

$$
\Phi\left(u_{i}\right)=\int_{-\infty}^{u_{i}} \frac{1}{\sqrt{2 \pi}} e^{-\frac{1}{2} x^{2}} d x
$$

\section{Appendix B. The General Form of $\Upsilon_{t}$}

Let $N$ denote the number of assets, and $N_{X}$ the number of explanatory variables. Define $A$ as a $N x\left(N+N_{X}\right)$ matrix whose first $N$ columns are an identity matrix. The last $N_{X}$ columns are the Hadamard product of a $N \mathrm{x} N_{X}$ matrix of coefficients $\theta$ and a matrix of explanatory regressors $X_{t}$

$$
A=\left[\begin{array}{ccccccc}
1 & 0 & \ldots & 0 & \theta_{11} X_{11, t} & \ldots & \theta_{1 N_{X}} X_{1 N_{X}, t} \\
0 & 1 & \ldots & 0 & \theta_{21} X_{21, t} & \ldots & \theta_{2 N_{X}} X_{2 N_{X}, t} \\
\vdots & \vdots & \ddots & 0 & \vdots & \vdots & \vdots \\
0 & 0 & \ldots & 1 & \theta_{N_{X} 1} X_{N_{X} 1, t} & \ldots & \theta_{N_{X} N_{X}} X_{N_{X} N_{X}, t}
\end{array}\right]
$$

The matrix $A$ is standardized by dividing each element by its row's root mean square

$$
\bar{A}_{i, j}=\frac{A_{i, j}}{\sqrt{\sum_{k=1}^{N+N_{X}} A_{i, k}^{2}}}=\frac{A_{i, j}}{\sqrt{1+\sum_{k=N+1}^{N+N_{X}} A_{i, k}^{2}}},
$$

and then $\Upsilon_{t}$ is given by

$$
\Upsilon_{t}=\bar{A} \bar{A}^{\top}
$$

These restrictions ensure that $\Upsilon_{t}$ is a proper correlation matrix. Up to a normalizing constant, this matrix will have as a typical off-diagonal element

$$
\Upsilon_{i j, t}=\sum_{k=1}^{N_{X}} \theta_{i k} \theta_{j k} X_{i k, t} X_{j k, t}, \quad i \neq j .
$$

This specification can easily accommodate for a time trend $\left(X_{i k, t}=t\right)$, a common positive factor $\left(X_{i k, t}=X_{j k, t}=\sqrt{F_{t}}\right)$, and asset specific variables, for example, volatilities $X_{i k, t}=\sigma_{i, t}$.

\section{Appendix C. Copula Correlation Moment Matching}

Equating the elements of $\Omega$ in the DAC model to the sample average $\frac{\frac{1}{T} \sum_{t=1}^{T} \bar{z}_{t}^{*} \bar{z}_{t}^{* \top}-\varphi_{\Gamma} \frac{1}{T} \sum_{t=1}^{T} \Upsilon_{t}}{1-\varphi_{\Gamma}}$ allows us to significantly reduce the number of parameters estimated via numerical optimization of the likelihood function. 
Recall that the DAC recursion is given by

$$
\Gamma_{t}=\left(1-\beta_{\Gamma}-\alpha_{\Gamma}\right)\left[\left(1-\varphi_{\Gamma}\right) \Omega+\varphi_{\Gamma} \Upsilon_{t}\right]+\beta_{\Gamma} \Gamma_{t-1}+\alpha_{\Gamma} \bar{z}_{t-1}^{*} \bar{z}_{t-1}^{*}
$$

where $\bar{z}_{i, t}^{*}=z_{i, t}^{*} \sqrt{\Gamma_{i i, t}}$. A circularity problem is apparent because we need $\Gamma_{i i, t}$ to estimate $\Omega$, which in turn is required to compute the time series of $\Gamma_{i i, t}$. Note however that $\Omega$ is a copula correlation matrix, so that $\Omega_{i i}=1$, for all $i$, and note also that only the diagonal elements of $\Gamma_{t}$ are needed to compute $\bar{z}_{i, t}^{*}$. Aielli (2009) therefore proposes to first compute equation (6.6) for the diagonal elements only, that is

$$
\Gamma_{i i, t}=\left(1-\alpha_{\Gamma}-\beta_{\Gamma}\right)+\beta_{\Gamma} \Gamma_{i i, t-1}+\alpha_{\Gamma}\left(\bar{z}_{i i, t-1}^{*}\right)^{2}
$$

for all $i$ and $t$. Having computed the $\Gamma_{i i, t}$, the sample correlation matrix of the $\bar{z}_{i, t}^{*}$ can be obtained which in turn yields $\widehat{\Omega}$, and the recursion in (6.6) can now be implemented replacing $\Omega$ by $\widehat{\Omega}$. 
Figure 1: Average Dynamic Copula Correlations for Developed, Emerging, and All Markets.

1989-2008

16 Developed Markets

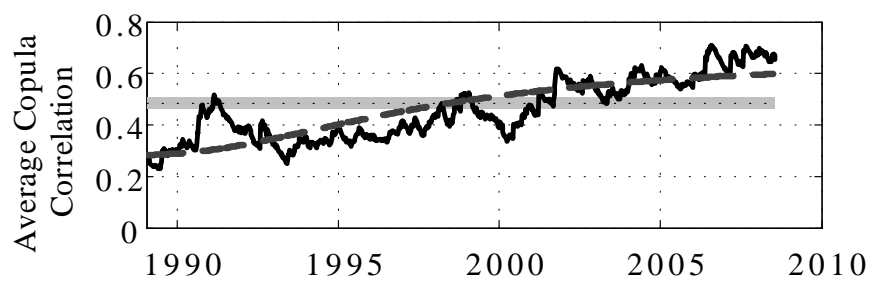

13 Emerging Markets, IFCG

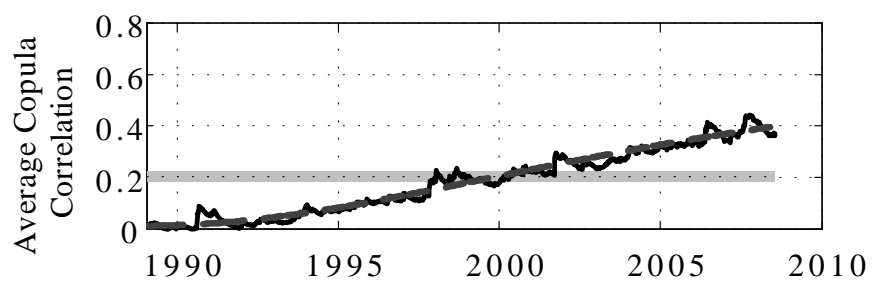

All 29 Markets

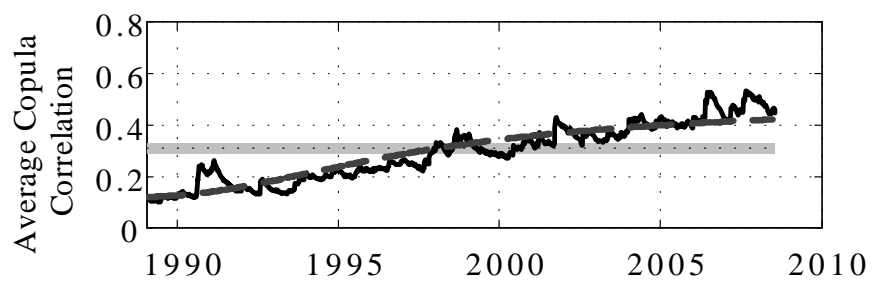

16 DMs vs 13 EMs Cross-Correlation

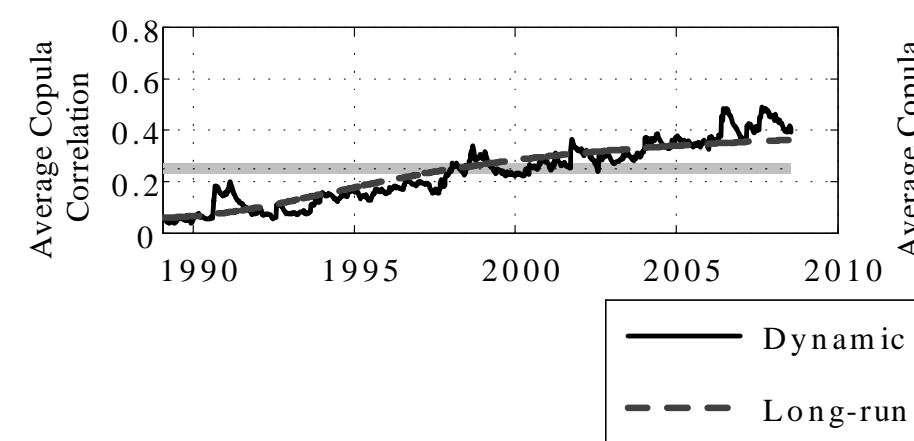

1995-2009

16 Developed Markets

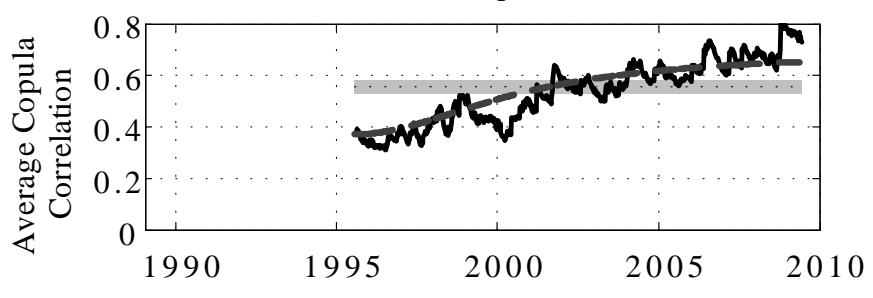

17 Emerging Markets, IFCI

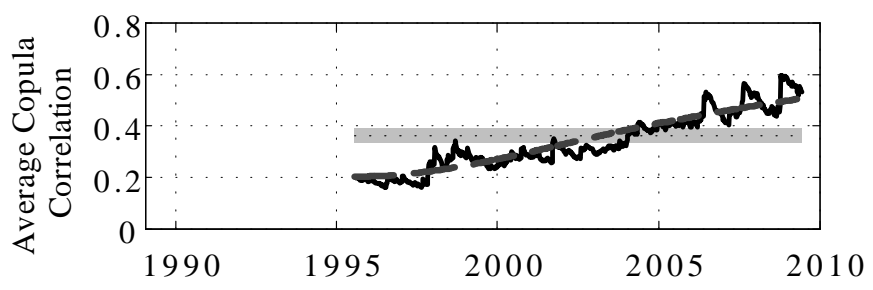

All 33 Markets

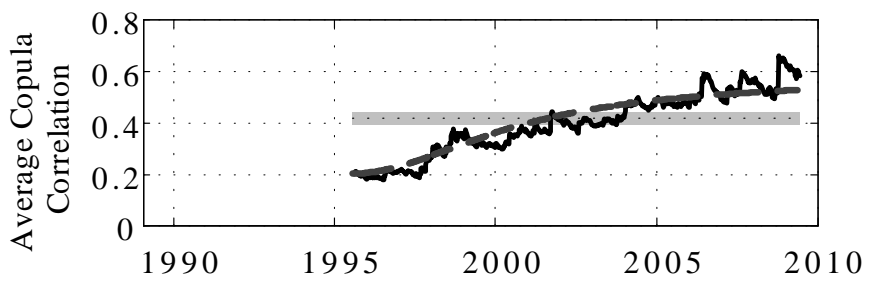

16 DMs vs 17 EMs Cross-Correlation

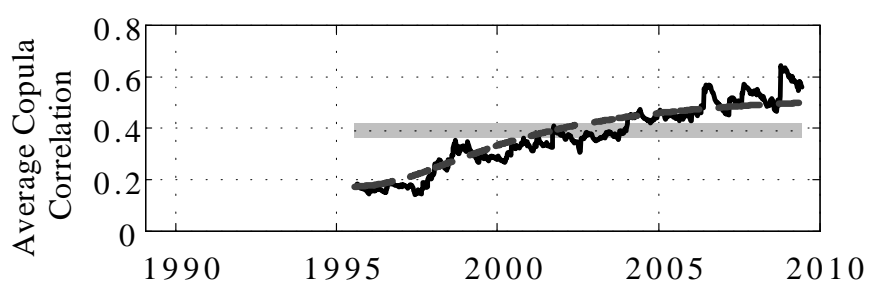

Notes to Figure: We report dynamic copula correlations, long run copula correlations and constant copula correlation averaged across countries along with a $90 \%$ bootstrap confidence interval. The left-side panels report on the period January 20, 1989 to July 25, 2008. The right-side panels report on the period July 21, 1995 to June 12, 2009. The top panels report on developed markets, the middle panels report on emerging markets, the third panels report on both, and the bottom panels report the average cross-correlations between developed and emerging markets. The copula correlations are computed from the DAC model. 
Figure 2: Average Dynamic Copula Correlations for Developed Markets. Various Sample Periods.
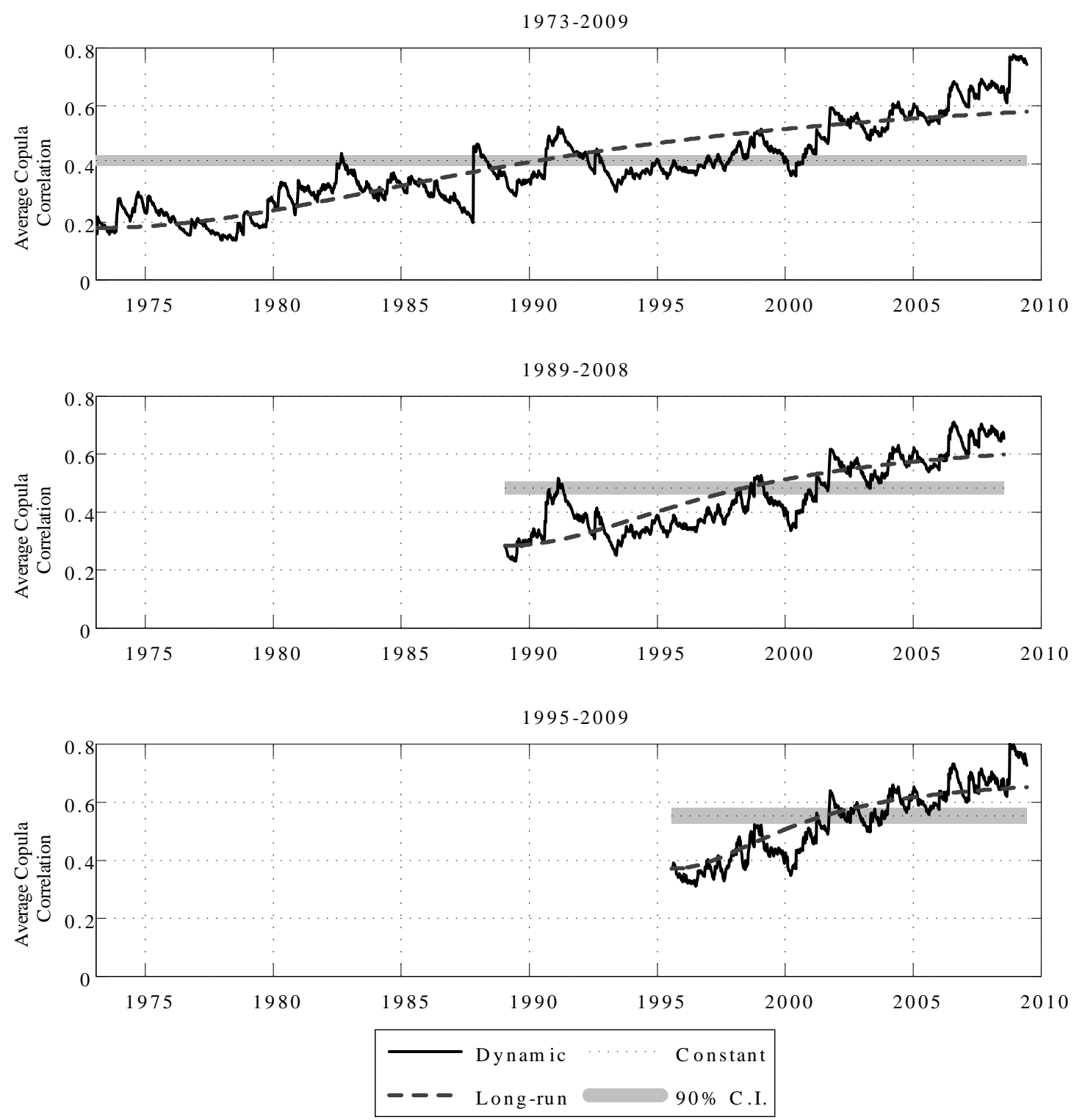

Notes to Figure: We report dynamic copula correlations, long run copula correlations and constant copula correlation averaged across developed markets along with a $90 \%$ bootstrap confidence interval. The top panel reports on the period January 26, 1973 to June 12, 2009. The middle panel reports on the period January 20, 1989 to July 25, 2008. The bottom panel reports on the period July 21, 1995 to June 12, 2009. The copula correlations are computed from the DAC model. 
Figure 3.A: Copula Correlations for Each Developed Market.
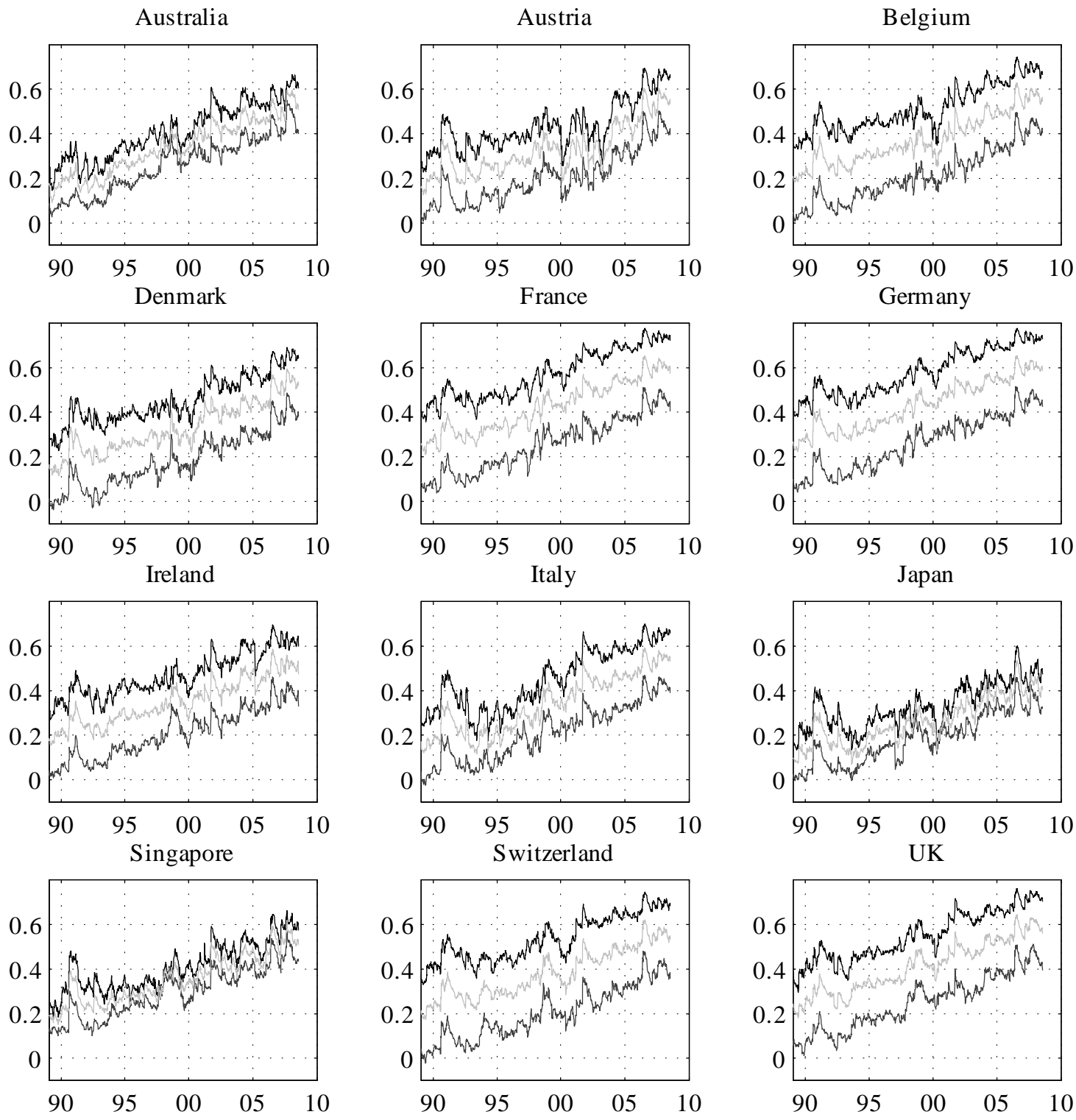

Notes to Figure: We report dynamic copula correlations for sixteen developed markets for the period January 20, 1989 to July 21, 2008. For each country, at each point in time we report: the average copula correlations with the fifteen other developed markets (black line), with the thirteen emerging markets (dark grey line), and with all the 28 other markets (light grey line). The copula correlations are computed from the DAC model. 
Figure 3.B: Copula Correlations for each Emerging Market.
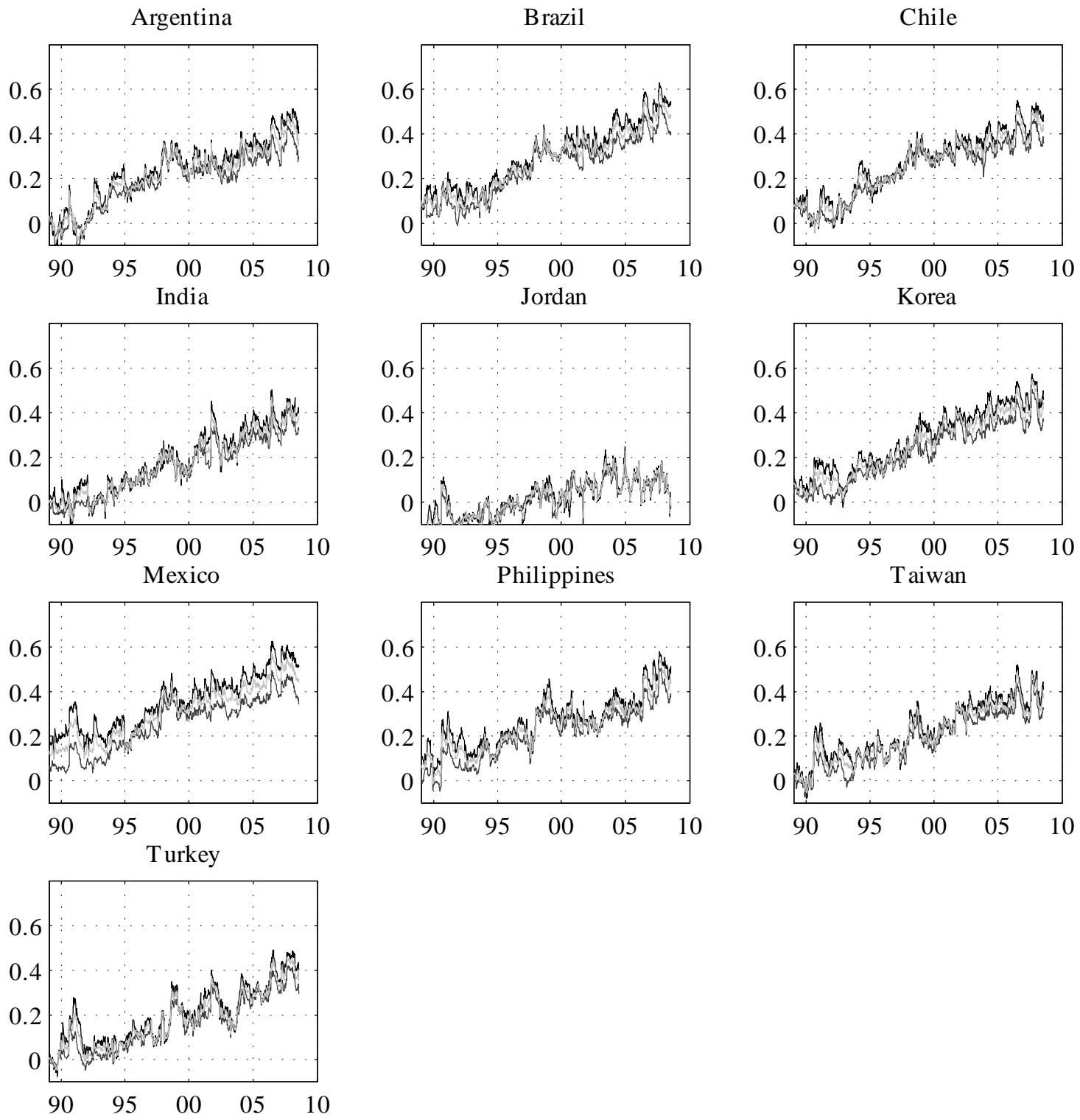

Notes to Figure: We report dynamic copula correlations for thirteen emerging markets for the period January 20, 1989 to July 25, 2008. For each country, at each point in time we report: the average copula correlations with sixteen developed markets (black line), with the twelve other emerging markets (dark grey line), and with all the 28 other markets (light grey line). The copula correlations are computed from the DAC model. 
Figure 4: Regional Copula Correlation Patterns.
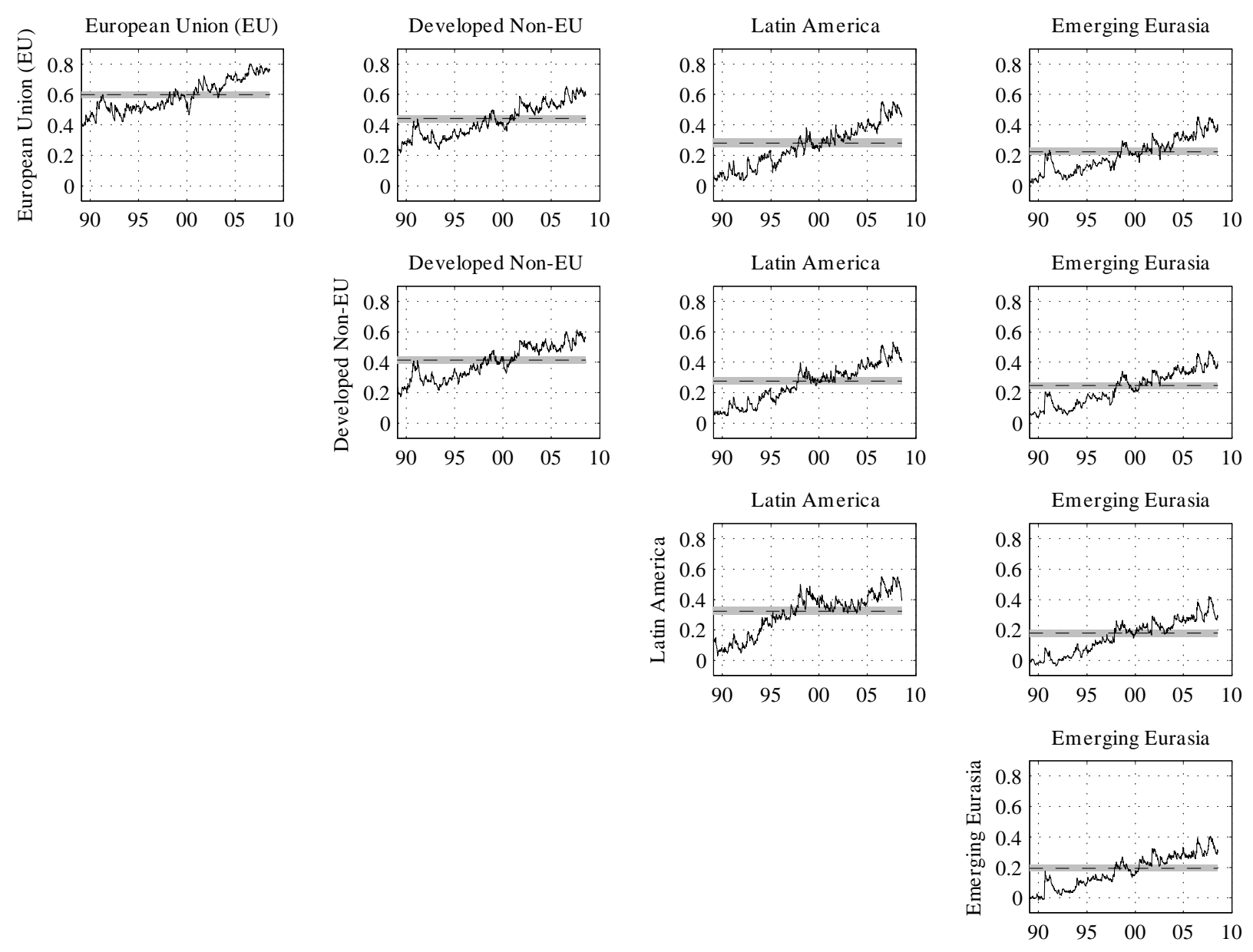

Notes to Figure: We use the DAC model to plot the average copula correlation within and across four regions. We also plot the constant copula correlation along with a $90 \%$ bootstrap confidence interval (dashed line with gray area). The European Union (EU) includes Austria, Belgium, Denmark, France, Germany, Ireland, Italy, Netherlands, and the UK. Developed Non-EU includes Australia, Canada, Hong Kong, Japan, Singapore, Switzerland, and the US. Latin America includes Argentina, Brazil, Chile, Colombia, and Mexico. Emerging Eurasia includes India, Jordan, Korea, Malaysia, Philippines, Taiwan, Thailand, and Turkey. 
Figure 5: Copula Correlation Range (90th and 10th Percentile).

Developed, Emerging, and All Markets.
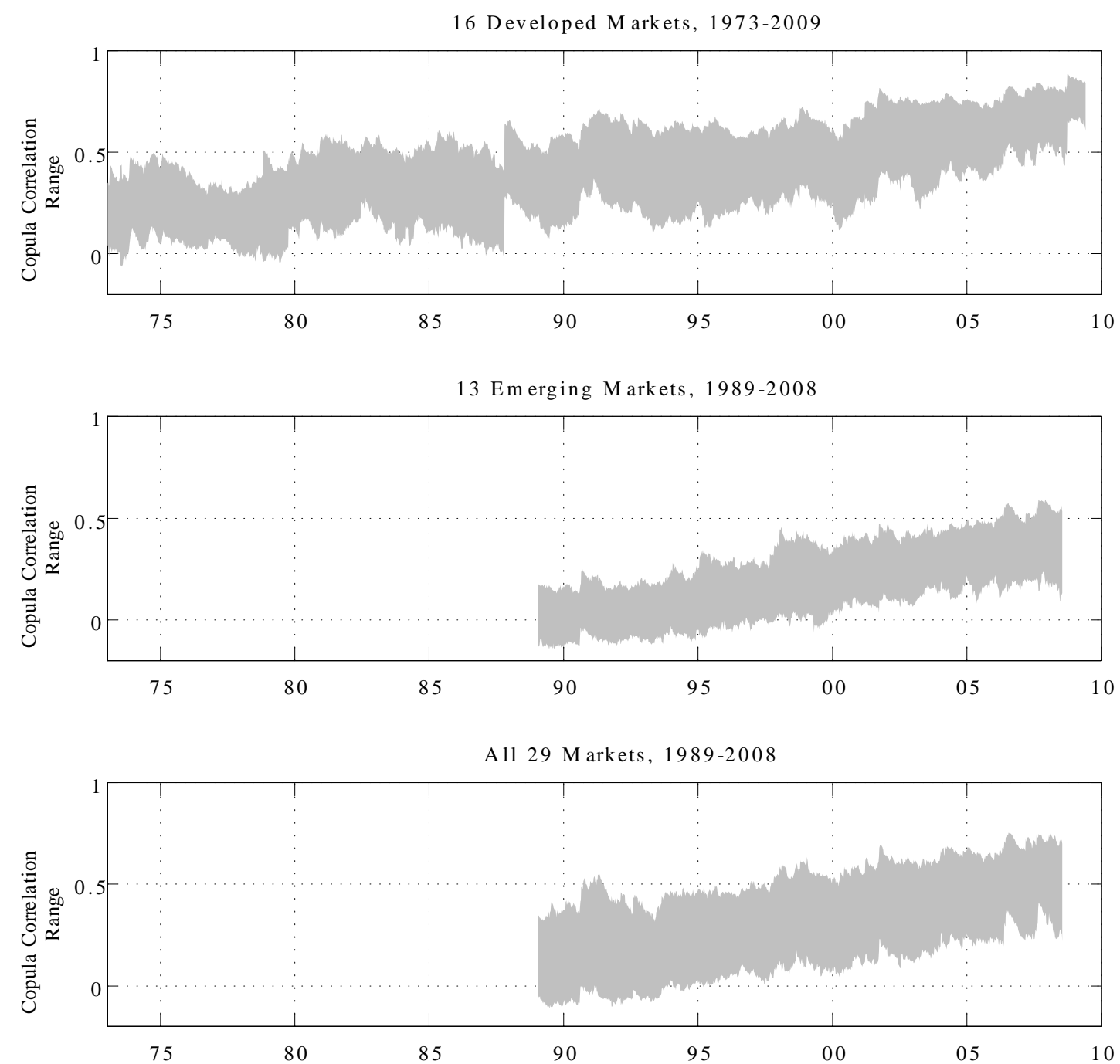

Notes to Figure: The shaded areas show the DAC correlation range between the 90th and 10th percentiles. The top panel reports on sixteen developed markets for the period January 26, 1973 to June 12, 2009. The middle panel reports on thirteen emerging markets for the period January 20, 1989 to July 25, 2008. The bottom panel reports on sixteen developed and thirteen emerging markets for the period January 20, 1989 to July 25, 2008. 
Figure 6: Conditional Diversification Benefits (CDB) using the DAC Model. Developed, Emerging, and All Markets.
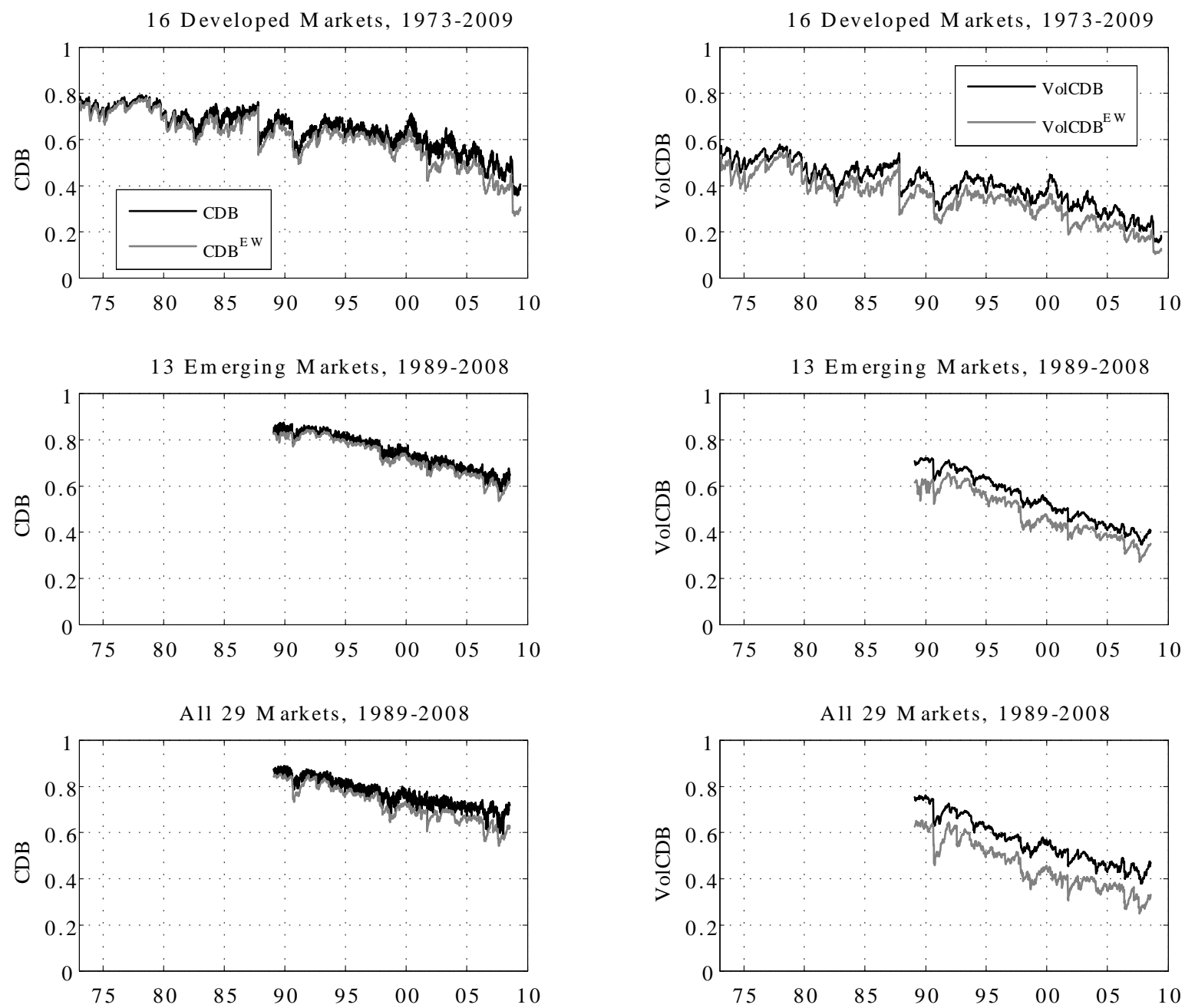

Notes to Figure: Each week and for each set of countries, we use the DAC model to compute the conditional diversification benefit $(C D B)$ in equation (4.1) in the left panels. In the right panels we use the volatility-based measure conditional diversification benefit $(\operatorname{Vol} C D B$ ) measure from equation (4.3). The superscript " $E W$ " refers to equal-weighted portfolios. 
Figure 7: Dynamic Average Bivariate Tail Dependence for the DAC Model.

16 Developed M arkets

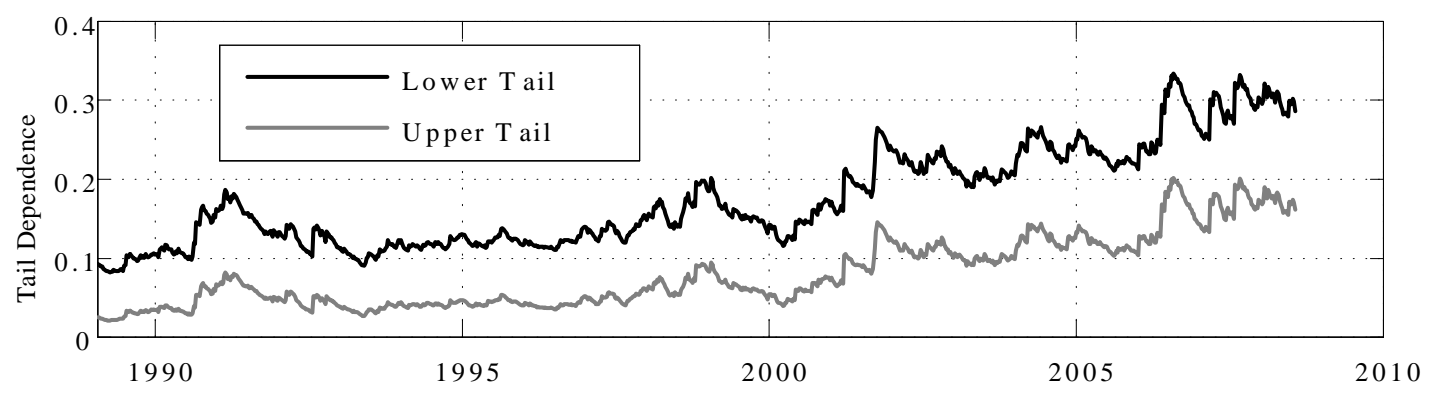

13 Emerging Markets

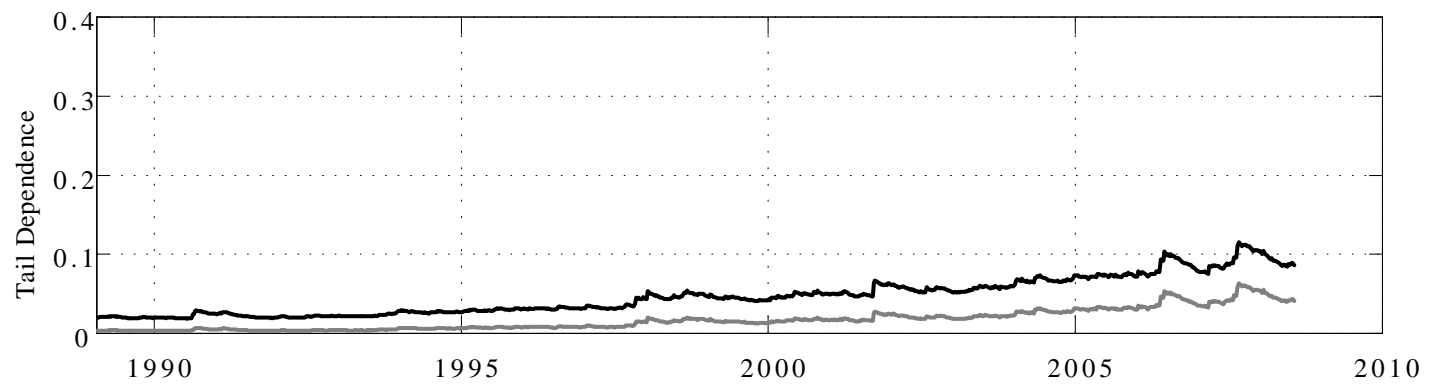

All 29 M arkets

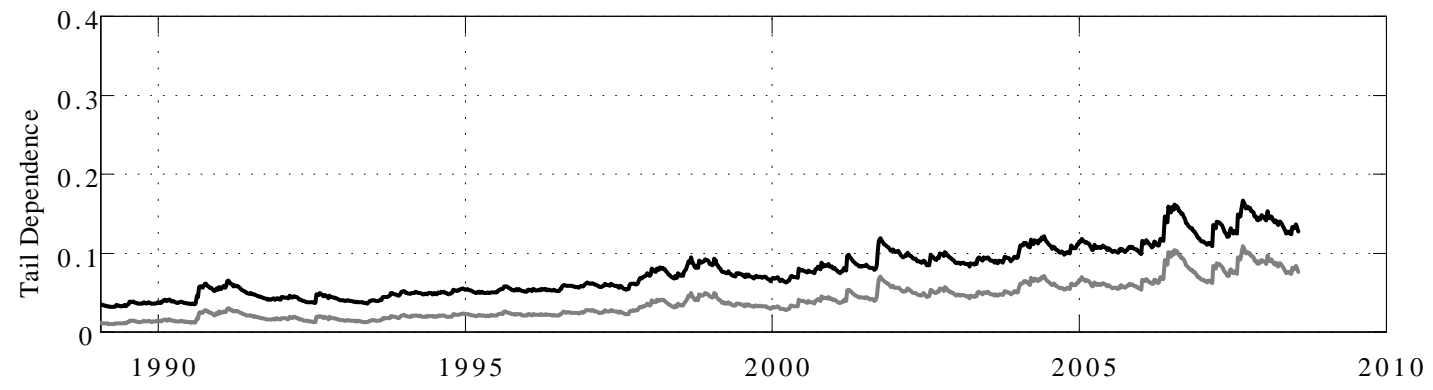

Notes to Figure: We report the average bivariate tail dependence for the DAC model. The black line denotes lower tail dependence and the gray line denotes upper tail dependence. The top panels report on sixteen developed markets, the middle panels report on thirteen emerging markets, and the bottom panels report on all 29 markets for the period January 20, 1989 to July 25, 2008. 
Figure 8: Empirical Threshold Correlations.
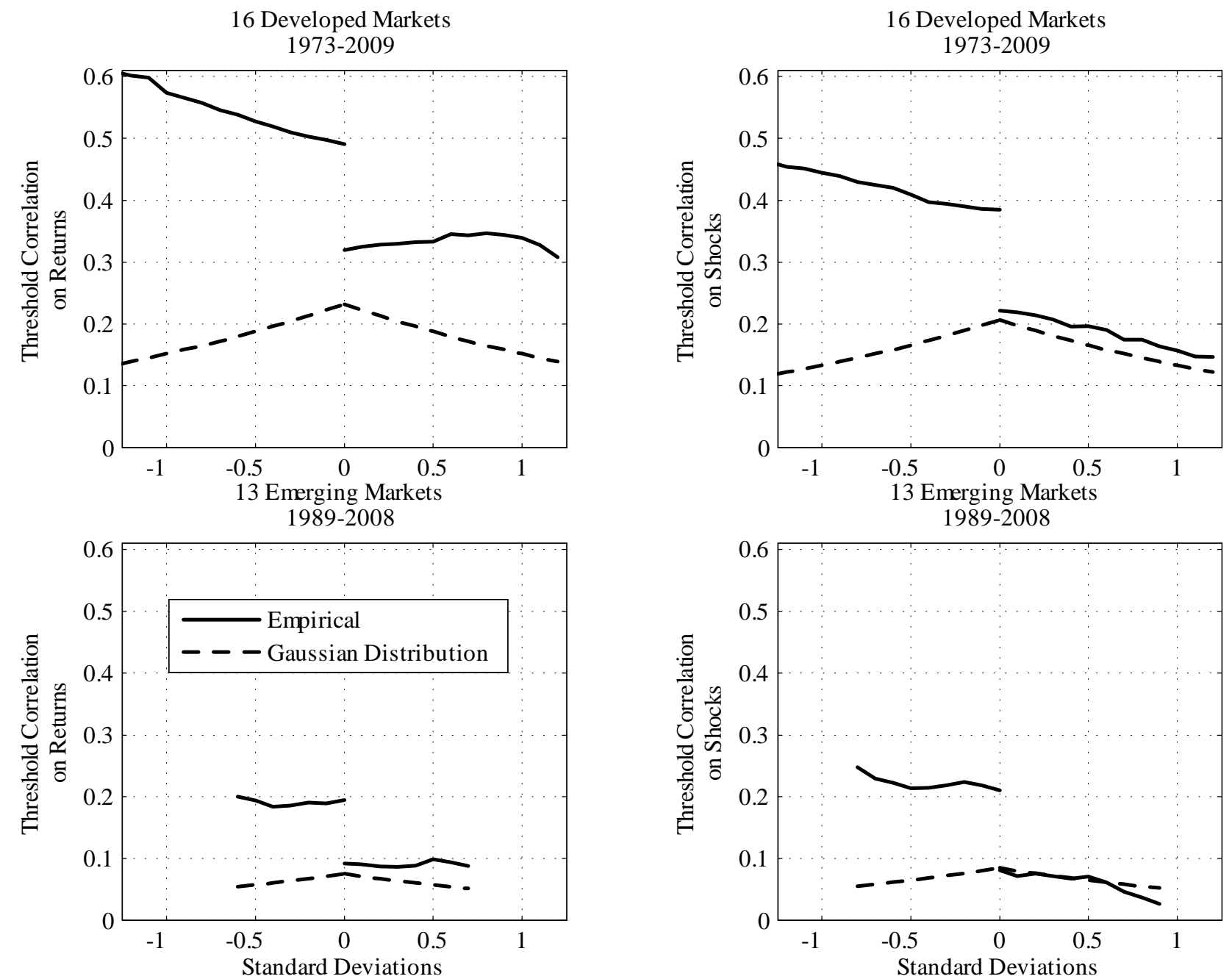

Notes to Figure: The top panels report threshold correlations for sixteen developed markets for the period January 26, 1973 to June 12, 2009. The bottom panels report the threshold correlations for thirteen emerging markets for the period January 20, 1989 to July 25, 2008. The left column reports the average pairwise threshold correlations computed on returns, which are standardized by their unconditional means and standard deviations. The right column presents the average pairwise threshold correlations computed on shocks, which are returns standardized using the AR-NGARCH model with skewed $t$ innovations. In each case, we compare the empirical threshold correlation (solid line) to the one implied by a normal distribution (dashed line) using the average linear correlation estimated from the data. 
Figure 9: Model Threshold Correlations.

16 Developed Markets

1973-2009
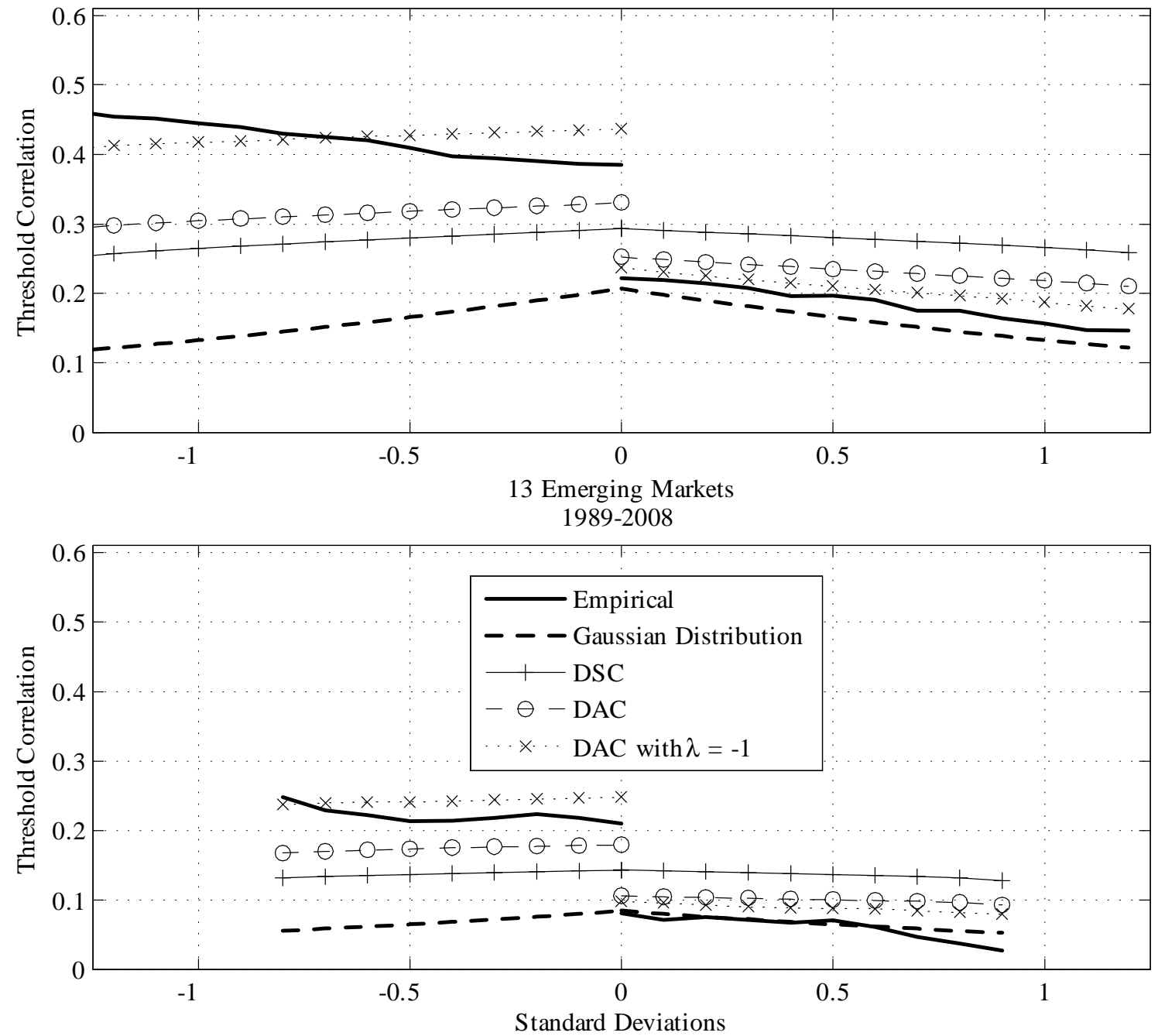

Notes to Figure: The top panel reports the average pairwise threshold correlations for sixteen developed markets for the period January 26, 1973 to June 12, 2009. The bottom panel reports the average pairwise threshold correlations for thirteen emerging markets for the period January 20, 1989 to July 25, 2008. We compute threshold correlations using the empirical AR-NGARCH residuals as well as threshold correlations based on generated data from three models: the multivariate Gaussian distribution, the DSC model, and the DAC model. The DAC model with $\lambda=-1$ calibrates the $\lambda$ parameter rather than using the empirical estimate. 
Figure 10: Monthly and Annual Model-Based Copula Correlations.

1989-2008

16 Developed Markets

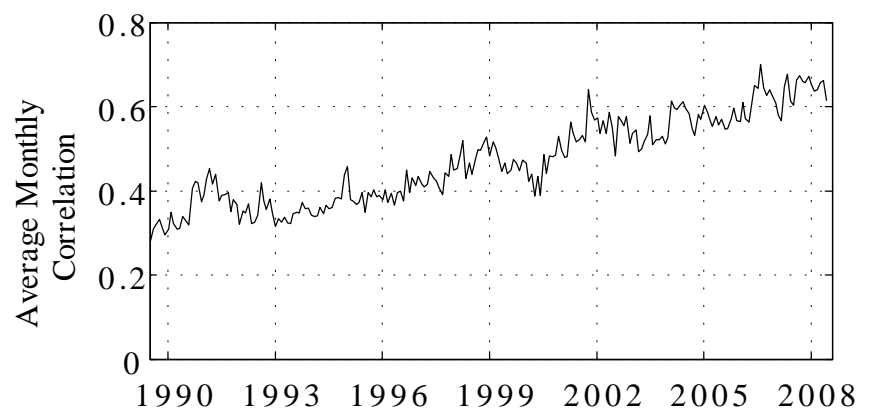

13 Emerging Markets

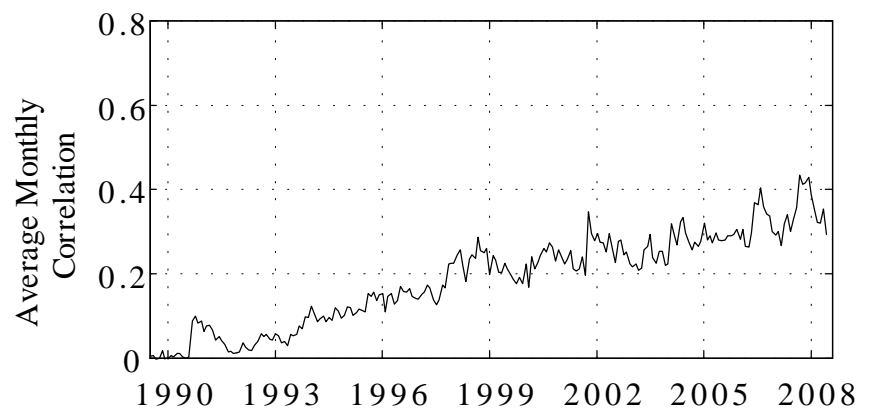

All 29 Markets

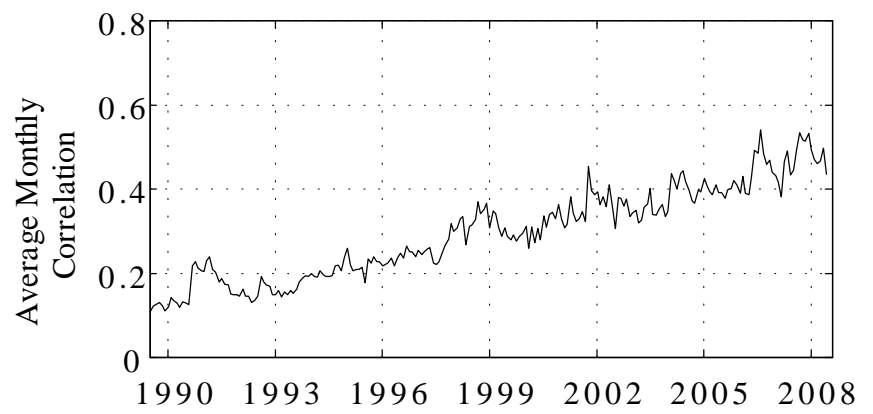

1989-2008

16 Developed Markets

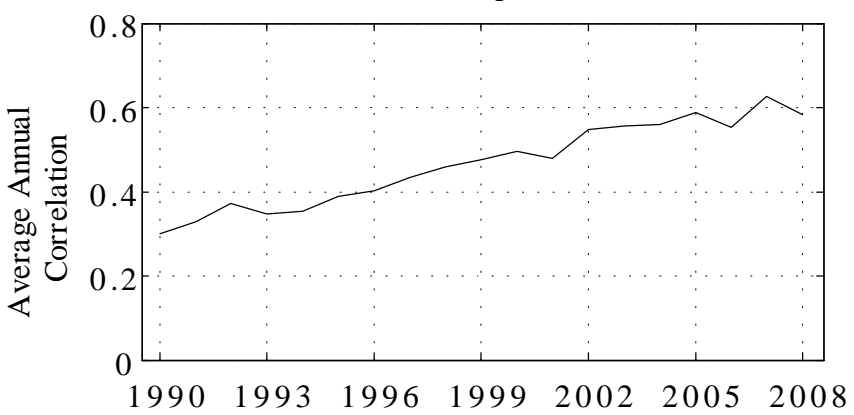

13 Emerging Markets

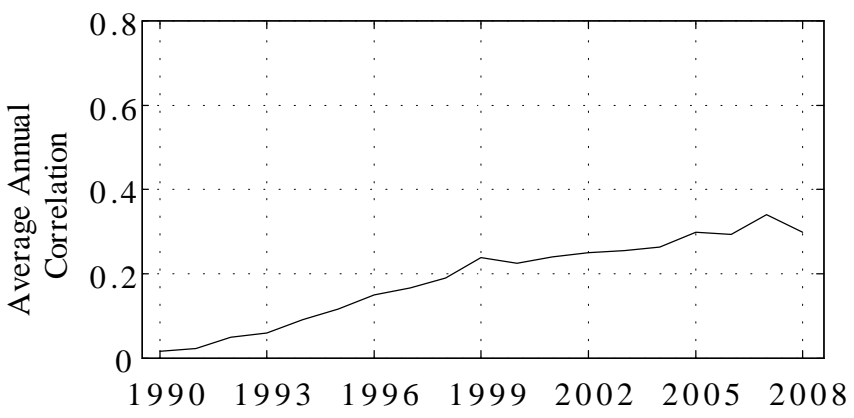

All 29 Markets

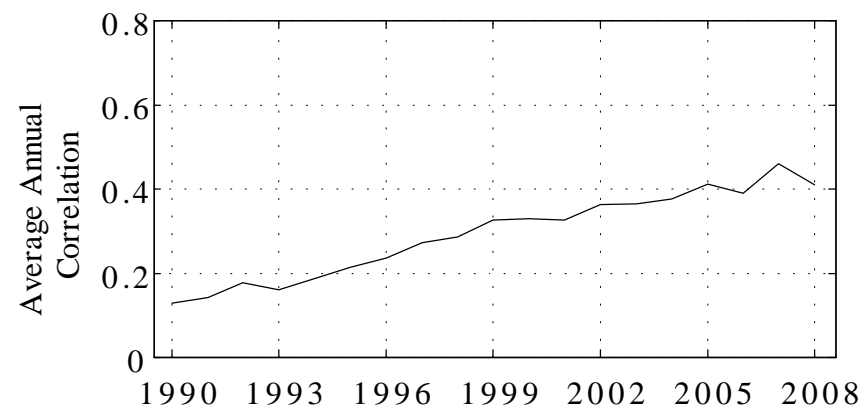

Notes to Figure: We report monthly and annual copula correlations averaged across countries simulated from the AR-NGARCH and DAC models. The left panels report monthly copula correlations, and the right panels report annual copula correlations. The top panels report on sixteen developed markets, the middle panels report on thirteen emerging markets, and the bottom panels report on sixteen developed and thirteen emerging markets for the period January 20, 1989 to July 25, 2008. 
Figure A.1: Contours of the Normal, $t$ and Skewed $t$ Copulas.
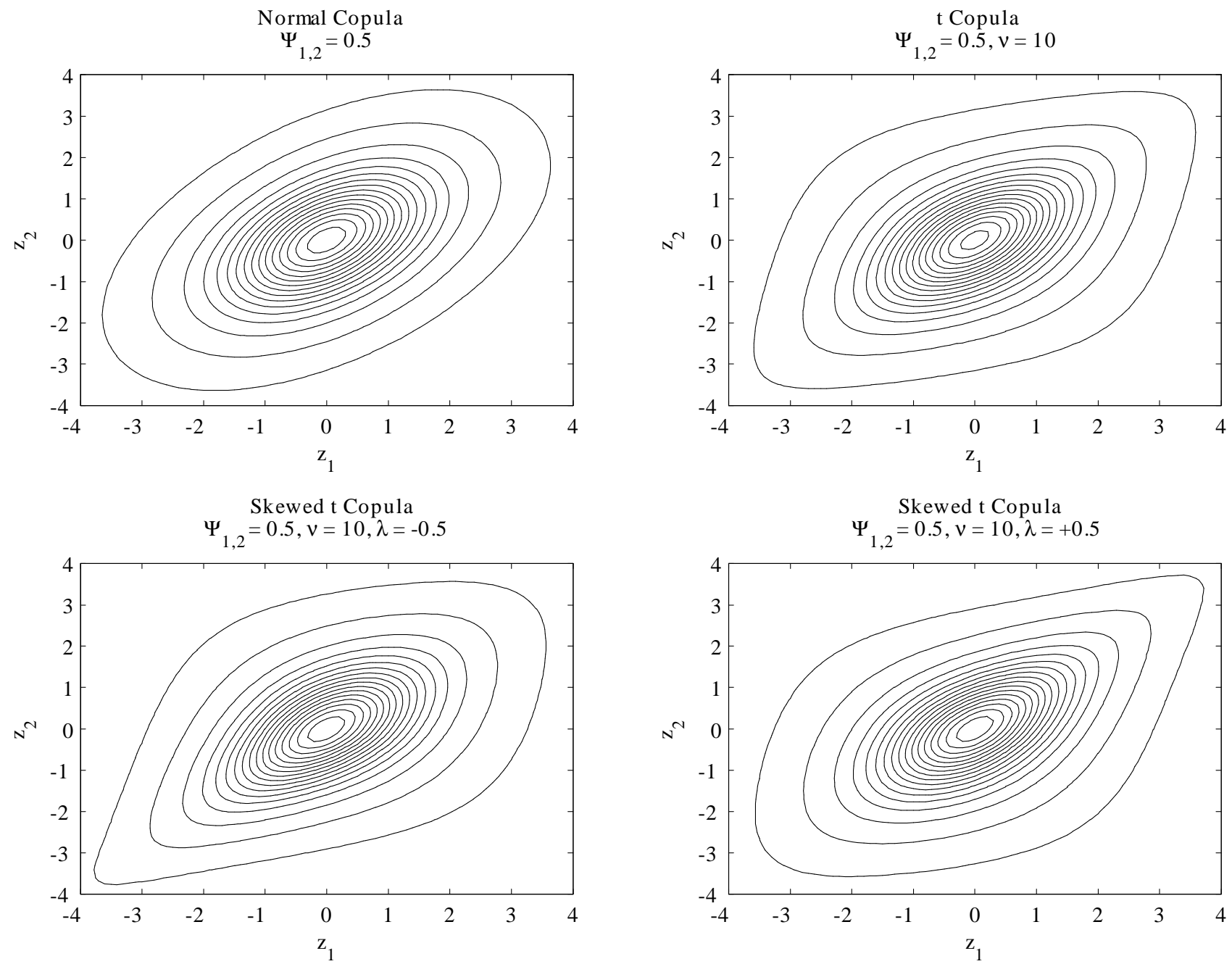

Notes to Figure: We plot the probability contours of four bivariate copula models assuming standard normal marginal distributions. The top left panel shows the normal copula with a copula correlation, $\Psi_{12}=0.5$. The top right panel shows the symmetric $t$ copula with $\Psi_{12}=0.5$ and degree of freedom, $\nu=10$. The bottom two panels show the new skewed $t$ copula where we keep $\Psi_{12}=0.5$ and $\nu=$ 10. The bottom left panel has an asymmetry parameter, $\lambda=-0.5$ and the bottom right panel has an asymmetry parameter, $\lambda=+0.5$. The probability levels for each contour are the kept the same across all four copula models. 
Table 1: Descriptive Statistics for Weekly Returns on 16 DM and 13 EM (IFCG).

January 1989 to July 2008.

\begin{tabular}{|c|c|c|c|c|c|c|c|}
\hline & $\begin{array}{c}\text { Annual } \\
\text { Mean (\%) }\end{array}$ & $\begin{array}{c}\text { Annual } \\
\text { Standard } \\
\text { Deviation } \\
(\%)\end{array}$ & Skewness & $\begin{array}{c}\text { Excess } \\
\text { Kurtosis }\end{array}$ & $\begin{array}{c}\text { 1st Order } \\
\text { Auto- } \\
\text { correlation }\end{array}$ & $\begin{array}{l}\text { LB(20) P- } \\
\text { Value on } \\
\text { Returns }\end{array}$ & $\begin{array}{c}\text { LB(20) P- } \\
\text { value on } \\
\text { Absolute } \\
\text { Returns }\end{array}$ \\
\hline \multicolumn{8}{|l|}{$\begin{array}{l}\text { Developed } \\
\text { Markets }\end{array}$} \\
\hline Australia & 13.21 & 17.13 & -0.301 & 1.94 & -0.010 & 0.2044 & 0.0000 \\
\hline Austria & 14.50 & 19.63 & -0.009 & 3.69 & 0.058 & 0.1364 & 0.0000 \\
\hline Belgium & 10.93 & 16.55 & -0.215 & 2.00 & 0.011 & 0.8316 & 0.0000 \\
\hline Canada & 13.24 & 16.30 & -0.489 & 2.35 & -0.001 & 0.1611 & 0.0000 \\
\hline Denmark & 14.55 & 17.83 & -0.308 & 2.11 & -0.045 & 0.2535 & 0.0000 \\
\hline France & 12.75 & 17.54 & -0.120 & 1.04 & 0.019 & 0.0632 & 0.0000 \\
\hline Germany & 11.88 & 18.66 & -0.231 & 1.65 & 0.018 & 0.7596 & 0.0000 \\
\hline Hong Kong & 16.71 & 24.41 & -0.355 & 3.11 & 0.030 & 0.0046 & 0.0000 \\
\hline Ireland & 13.30 & 18.89 & -0.286 & 1.74 & 0.011 & 0.2749 & 0.0000 \\
\hline Italy & 9.18 & 20.99 & 0.189 & 3.32 & 0.037 & 0.1494 & 0.0000 \\
\hline Japan & 1.45 & 22.28 & 0.339 & 1.56 & -0.068 & 0.0307 & 0.0000 \\
\hline Netherlands & 13.26 & 16.62 & -0.300 & 3.00 & 0.006 & 0.3920 & 0.0000 \\
\hline Singapore & 11.16 & 20.66 & -0.255 & 5.15 & -0.009 & 0.0010 & 0.0000 \\
\hline Switzerland & 13.70 & 16.42 & 0.002 & 2.05 & -0.012 & 0.5579 & 0.0001 \\
\hline United Kingdom & 11.37 & 15.54 & 0.060 & 1.80 & -0.027 & 0.6799 & 0.0019 \\
\hline United States & 11.71 & 15.15 & -0.434 & 3.46 & -0.102 & 0.0005 & 0.0000 \\
\hline Average & 12.06 & 18.41 & -0.170 & 2.50 & -0.005 & 0.2813 & 0.0001 \\
\hline \multicolumn{8}{|l|}{$\begin{array}{l}\text { Emerging } \\
\text { Markets }\end{array}$} \\
\hline Argentina & 29.44 & 51.18 & 0.804 & 12.22 & -0.009 & 0.0001 & 0.0000 \\
\hline Brazil & 29.85 & 46.23 & -0.246 & 2.22 & 0.045 & 0.3060 & 0.0000 \\
\hline Chile & 19.43 & 21.18 & 0.008 & 1.30 & 0.167 & 0.0000 & 0.0000 \\
\hline Colombia & 22.66 & 26.51 & 0.339 & 6.29 & 0.132 & 0.0000 & 0.0000 \\
\hline India & 15.24 & 27.89 & -0.027 & 2.05 & 0.078 & 0.0494 & 0.0000 \\
\hline Jordan & 15.86 & 18.21 & 0.195 & 5.42 & 0.083 & 0.0071 & 0.0000 \\
\hline Korea & 10.18 & 36.43 & 0.045 & 8.54 & -0.089 & 0.0001 & 0.0000 \\
\hline Malaysia & 10.16 & 28.64 & 1.394 & 24.02 & 0.013 & 0.0000 & 0.0000 \\
\hline Mexico & 20.99 & 28.02 & -0.387 & 3.72 & 0.108 & 0.0018 & 0.0000 \\
\hline Philippines & 7.21 & 28.57 & -0.393 & 4.97 & 0.085 & 0.0002 & 0.0000 \\
\hline Taiwan & 7.15 & 32.82 & 0.318 & 3.96 & -0.007 & 0.0232 & 0.0000 \\
\hline Thailand & 10.65 & 35.90 & 0.250 & 3.98 & 0.019 & 0.0000 & 0.0000 \\
\hline Turkey & 31.02 & 55.59 & 0.178 & 5.27 & -0.018 & 0.1912 & 0.0000 \\
\hline Average & 17.68 & 33.63 & 0.191 & 6.46 & 0.047 & 0.0445 & 0.0000 \\
\hline
\end{tabular}

Notes to Table: We report the first four sample moments and the first order autocorrelation of the 16 DM and 13 EM (IFCG) returns. We also report the p-value from a Ljung-Box test that the first 20 autocorrelations are zero for returns and absolute returns. The sample period is from January 20, 1989 to July 25, 2008. 
Table 2: Parameter Estimates from NGARCH(1,1) on 16 DM and 13 EM (IFCG). January 1989 to July 2008.

\begin{tabular}{|c|c|c|c|c|c|c|c|c|}
\hline & $\alpha$ & $\beta$ & $\gamma$ & $\begin{array}{c}\text { Variance } \\
\text { Persistence } \\
\end{array}$ & e & $\chi$ & $\begin{array}{c}\mathrm{LB}(20) \mathrm{P}- \\
\text { Value on } \\
\text { Residuals }\end{array}$ & $\begin{array}{c}\text { LB(20) P- } \\
\text { Value on } \\
\text { Absolute } \\
\text { Residuals } \\
\end{array}$ \\
\hline \multicolumn{9}{|c|}{ Developed Markets } \\
\hline Australia & 0.053 & 0.832 & 0.697 & 0.912 & 11.354 & -0.146 & 0.653 & 0.784 \\
\hline Austria & 0.087 & 0.888 & -0.134 & 0.977 & 9.635 & -0.038 & 0.434 & 0.327 \\
\hline Belgium & 0.139 & 0.608 & 0.695 & 0.814 & 10.220 & -0.097 & 0.827 & 0.262 \\
\hline Canada & 0.092 & 0.854 & 0.339 & 0.957 & 10.026 & -0.174 & 0.611 & 0.782 \\
\hline Denmark & 0.052 & 0.935 & -0.108 & 0.988 & 7.203 & -0.042 & 0.322 & 0.551 \\
\hline France & 0.067 & 0.717 & 1.190 & 0.878 & 22.179 & -0.098 & 0.122 & 0.396 \\
\hline Germany & 0.112 & 0.787 & 0.499 & 0.926 & 10.600 & -0.107 & 0.810 & 0.186 \\
\hline Hong Kong & 0.092 & 0.856 & 0.445 & 0.966 & 8.158 & -0.076 & 0.228 & 0.762 \\
\hline Ireland & 0.048 & 0.864 & 1.008 & 0.961 & 8.885 & -0.110 & 0.471 & 0.597 \\
\hline Italy & 0.098 & 0.828 & 0.345 & 0.937 & 8.624 & -0.067 & 0.442 & 0.083 \\
\hline Japan & 0.080 & 0.824 & 0.708 & 0.944 & 7.492 & 0.079 & 0.395 & 0.399 \\
\hline Netherlands & 0.123 & 0.726 & 0.655 & 0.903 & 12.938 & -0.223 & 0.793 & 0.683 \\
\hline Singapore & 0.082 & 0.880 & 0.457 & 0.979 & 5.813 & -0.027 & 0.465 & 0.888 \\
\hline Switzerland & 0.089 & 0.657 & 0.862 & 0.812 & 9.359 & -0.059 & 0.390 & 0.791 \\
\hline United Kingdom & 0.040 & 0.666 & 2.099 & 0.884 & 12.804 & -0.078 & 0.839 & 0.413 \\
\hline United States & 0.095 & 0.820 & 0.738 & 0.966 & 13.316 & -0.186 & 0.387 & 0.414 \\
\hline Average & 0.084 & 0.796 & 0.656 & 0.925 & 10.538 & -0.091 & 0.512 & 0.520 \\
\hline \multicolumn{9}{|c|}{ Emerging Markets } \\
\hline Argentina & 0.192 & 0.772 & 0.202 & 0.971 & 7.969 & -0.001 & 0.766 & 0.412 \\
\hline Brazil & 0.083 & 0.898 & 0.281 & 0.987 & 9.492 & -0.116 & 0.882 & 0.247 \\
\hline Chile & 0.084 & 0.872 & 0.084 & 0.957 & 10.054 & 0.060 & 0.553 & 0.915 \\
\hline Colombia & 0.191 & 0.682 & -0.080 & 0.875 & 4.381 & 0.053 & 0.488 & 0.575 \\
\hline India & 0.118 & 0.811 & 0.184 & 0.933 & 9.206 & -0.036 & 0.186 & 0.136 \\
\hline Jordan & 0.033 & 0.933 & -0.838 & 0.989 & 3.971 & 0.214 & 0.461 & 0.091 \\
\hline Korea & 0.091 & 0.885 & 0.334 & 0.986 & 9.351 & 0.053 & 0.688 & 0.500 \\
\hline Malaysia & 0.089 & 0.902 & 0.153 & 0.993 & 5.561 & 0.011 & 0.984 & 0.623 \\
\hline Mexico & 0.108 & 0.749 & 0.803 & 0.927 & 11.294 & -0.103 & 0.926 & 0.588 \\
\hline Philippines & 0.065 & 0.899 & 0.414 & 0.975 & 5.667 & -0.003 & 0.848 & 0.437 \\
\hline Taiwan & 0.112 & 0.837 & 0.459 & 0.972 & 7.570 & -0.007 & 0.587 & 0.733 \\
\hline Thailand & 0.100 & 0.860 & 0.401 & 0.976 & 7.507 & 0.074 & 0.985 & 0.186 \\
\hline Turkey & 0.072 & 0.884 & -0.060 & 0.956 & 5.439 & 0.076 & 0.380 & 0.218 \\
\hline Average & 0.103 & 0.845 & 0.180 & 0.961 & 7.497 & 0.021 & 0.672 & 0.435 \\
\hline
\end{tabular}

Notes to Table: We report parameter estimates and residual diagnostics for the $\operatorname{NGARCH}(1,1)$ models. The sample period for 16 DM and 13 EM (IFCG) weekly returns is from January 20, 1989 to July 25, 2008. The conditional mean is modeled by an AR(2) model. The coefficients from the AR models are not shown. The constant term in the GARCH model is fixed by variance targeting. 
Table 3: Parameter Estimates for Dynamic Asymmetric t Copula (DAC) Models. Developed, Emerging and All Markets.

\begin{tabular}{|c|c|c|c|c|c|c|c|c|}
\hline & $\alpha_{\Gamma}$ & $\beta_{\Gamma}$ & $\begin{array}{l}\text { Dependence } \\
\text { Persistence }\end{array}$ & $\varphi_{\Gamma} \mathrm{Y}_{\mathrm{DM}, \mathbf{T}}$ & $\varphi_{\Gamma} \mathrm{Y}_{\mathrm{EM}, \mathbf{T}}$ & $v$ & $\lambda$ & $\begin{array}{l}\text { Composite } \\
\text { Likelihood } \\
\end{array}$ \\
\hline & & & Weekly Retu & ns, Januar & $26,1973 \mathrm{tc}$ & une 12,2 & & \\
\hline 16 Developed Markets & $\begin{array}{c}0.026 \\
(0.004)\end{array}$ & $\begin{array}{c}0.963 \\
(0.008)\end{array}$ & 0.988 & $\begin{array}{c}0.3877 \\
(0.1950)\end{array}$ & - & $\begin{array}{c}15.19 \\
\left(\begin{array}{c}2.44)\end{array}\right)\end{array}$ & $\begin{array}{c}-0.38 \\
(0.12)\end{array}$ & 31,349 \\
\hline No Dynamics & - & - & - & $\begin{array}{c}0.3026 \\
(0.0392)\end{array}$ & - & $\begin{array}{c}8.44 \\
(0.00)\end{array}$ & $\begin{array}{l}-0.33 \\
(0.11)\end{array}$ & 28,631 \\
\hline & & $\underline{B:} \mathbf{U}$ & eekly IFCG F & eturns, Jan & lary 20,198 & o July 25 & 008 & \\
\hline 16 Developed Markets & $\begin{array}{c}0.030 \\
(0.008)\end{array}$ & $\begin{array}{c}0.954 \\
(0.026)\end{array}$ & 0.984 & $\begin{array}{c}0.3018 \\
(0.1245)\end{array}$ & - & $\begin{array}{c}17.65 \\
(0.09)\end{array}$ & $\begin{array}{c}-0.48 \\
(0.10)\end{array}$ & 22,131 \\
\hline No Dynamics & - & - & - & $\begin{array}{c}0.2522 \\
(0.0526)\end{array}$ & - & $\begin{array}{c}10.79 \\
(0.01)\end{array}$ & $\begin{array}{c}-0.26 \\
(0.15)\end{array}$ & 20,872 \\
\hline 13 Emerging Markets & $\begin{array}{c}0.019 \\
(0.005)\end{array}$ & $\begin{array}{c}0.935 \\
(0.030)\end{array}$ & 0.953 & - & $\begin{array}{c}0.3743 \\
(0.0553)\end{array}$ & $\begin{array}{c}22.36 \\
(0.02)\end{array}$ & $\begin{array}{c}-0.49 \\
(0.22)\end{array}$ & 3,192 \\
\hline No Dynamics & - & - & - & - & $\begin{array}{c}0.3765 \\
(0.0334)\end{array}$ & $\begin{array}{c}19.55 \\
(1.51)\end{array}$ & $\begin{array}{c}-0.30 \\
(0.09)\end{array}$ & 3,061 \\
\hline All 29 Markets & $\begin{array}{c}0.026 \\
(0.007)\end{array}$ & $\begin{array}{c}0.937 \\
(0.041)\end{array}$ & 0.963 & $\begin{array}{c}0.2862 \\
(0.0557)\end{array}$ & $\begin{array}{c}0.3009 \\
(0.0708)\end{array}$ & $\begin{array}{c}21.83 \\
(0.02)\end{array}$ & $\begin{array}{c}-0.41 \\
(0.14)\end{array}$ & 36,390 \\
\hline No Dynamics & - & - & - & $\begin{array}{c}0.2620 \\
(0.0184)\end{array}$ & $\begin{array}{c}0.2628 \\
(0.0260)\end{array}$ & $\begin{array}{c}18.83 \\
(0.14)\end{array}$ & $\begin{array}{c}-0.50 \\
(0.00)\end{array}$ & 34,248 \\
\hline & & & Weekly IFCI & Returns, Ju & y 21, 1995 & June 12, 2 & & \\
\hline 16 Developed Markets & $\begin{array}{c}0.040 \\
(0.009)\end{array}$ & $\begin{array}{c}0.925 \\
(0.018)\end{array}$ & 0.965 & $\begin{array}{c}0.2679 \\
(0.1206)\end{array}$ & - & $\begin{array}{c}18.61 \\
\left(\begin{array}{c}0.01)\end{array}\right.\end{array}$ & $\begin{array}{c}-0.56 \\
(0.40)\end{array}$ & 21,213 \\
\hline No Dynamics & - & - & - & $\begin{array}{c}0.2829 \\
(0.0895)\end{array}$ & - & $\begin{array}{c}16.23 \\
(3.11)\end{array}$ & $\begin{array}{c}-0.73 \\
(0.05)\end{array}$ & 20,535 \\
\hline 17 Emerging Markets & $\begin{array}{c}0.025 \\
(0.006)\end{array}$ & $\begin{array}{c}0.920 \\
(0.025)\end{array}$ & 0.945 & - & $\begin{array}{c}0.2891 \\
(0.0537)\end{array}$ & $\begin{array}{c}12.80 \\
(0.01)\end{array}$ & $\begin{array}{c}-0.45 \\
(0.10)\end{array}$ & 9,181 \\
\hline No Dynamics & - & - & - & - & $\begin{array}{c}0.3170 \\
(0.0458)\end{array}$ & $\begin{array}{c}19.20 \\
(0.14)\end{array}$ & $\begin{array}{c}-0.45 \\
(0.27)\end{array}$ & 8,844 \\
\hline All 33 Markets & $\begin{array}{c}0.026 \\
(0.011)\end{array}$ & $\begin{array}{c}0.924 \\
(0.101)\end{array}$ & 0.950 & $\begin{array}{c}0.3200 \\
(0.0551)\end{array}$ & $\begin{array}{c}0.2985 \\
(0.1571)\end{array}$ & $\begin{array}{c}12.16 \\
(0.05)\end{array}$ & $\begin{array}{c}-0.40 \\
(0.11)\end{array}$ & 52,143 \\
\hline No Dynamics & - & - & - & $\begin{array}{c}0.3814 \\
(0.0814)\end{array}$ & $\begin{array}{c}0.2890 \\
(0.0944)\end{array}$ & $\begin{array}{c}13.77 \\
\left(\begin{array}{c}2.60)\end{array}\right.\end{array}$ & $\begin{array}{c}-0.61 \\
(0.09)\end{array}$ & 50,593 \\
\hline
\end{tabular}

Notes to Table: We report parameter estimates for the DAC models for the 16 developed markets, 13 emerging markets (IFCG), 17 emerging markets (IFCI) and all markets for different sample periods. We also report the special case of constant copula correlation $\left(\beta_{\Gamma}=0\right.$ and $\left.\alpha_{\Gamma}=0\right)$. The standard errors in parentheses are computed using the method in Engle, Shephard and Sheppard (2008). 
Table 4: Parameter Estimates for Dynamic Symmetric t Copula (DSC) Models. Developed, Emerging and All Markets.

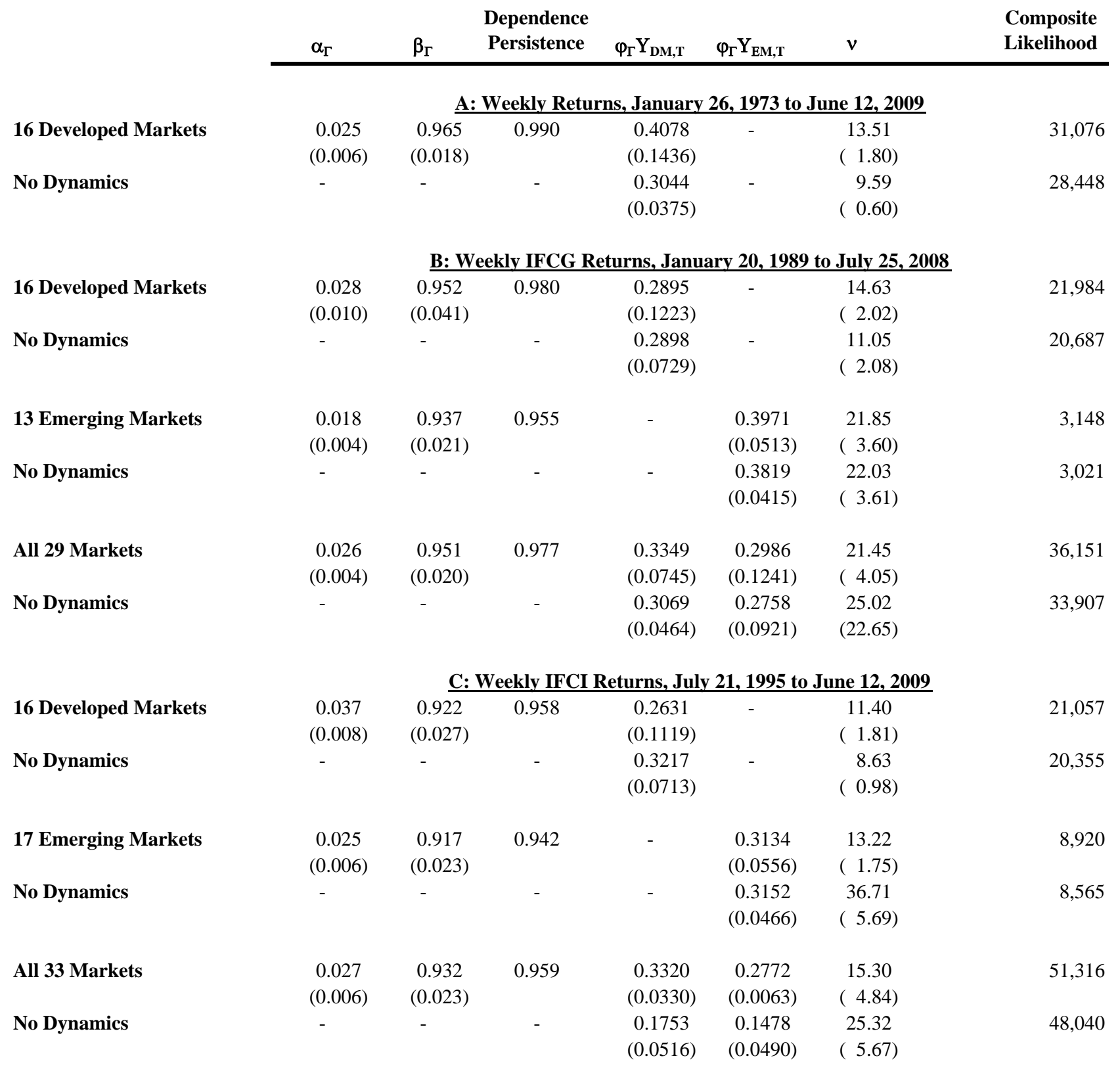

Notes to Table: We report parameter estimates for the DSC models for the 16 developed markets, 13 emerging markets (IFCG), 17 emerging markets (IFCI) and all markets for different sample periods. We also report the special case of constant copula correlations $\left(\beta_{\Gamma}=0\right.$ and $\left.\alpha_{\Gamma}=0\right)$. The standard errors in parentheses are computed using the method in Engle, Shephard and Sheppard (2008). 
Table 5: Parameter Estimates for Dynamic Normal Copula (DNC) Models. Developed, Emerging and All Markets.

\begin{tabular}{|c|c|c|c|c|c|c|}
\hline & $\alpha_{\Gamma}$ & $\beta_{\Gamma}$ & $\begin{array}{l}\text { Dependence } \\
\text { Persistence }\end{array}$ & $\varphi_{\Gamma} \mathrm{Y}_{\mathrm{DM}, \mathrm{T}}$ & $\varphi_{\Gamma} \mathrm{Y}_{\mathrm{EM}, \mathrm{T}}$ & $\begin{array}{l}\text { Composite } \\
\text { Likelihood }\end{array}$ \\
\hline & \multicolumn{6}{|c|}{ A: Weekly Returns, January 26, 1973 to June 12, 2009} \\
\hline 16 Developed Markets & $\begin{array}{c}0.023 \\
(0.005)\end{array}$ & $\begin{array}{c}0.967 \\
(0.010)\end{array}$ & 0.990 & $\begin{array}{c}0.5085 \\
(0.0669)\end{array}$ & - & 30,248 \\
\hline No Dynamics & - & - & - & $\begin{array}{c}0.3281 \\
(0.0365)\end{array}$ & - & 27,549 \\
\hline & \multicolumn{6}{|c|}{ B: Weekly IFCG Returns, January 20, 1989 to July 25, 2008} \\
\hline 16 Developed Markets & $\begin{array}{c}0.030 \\
(0.012)\end{array}$ & $\begin{array}{c}0.960 \\
(0.024)\end{array}$ & 0.990 & $\begin{array}{c}0.3099 \\
(0.1313)\end{array}$ & - & 21,651 \\
\hline No Dynamics & - & - & - & $\begin{array}{c}0.2795 \\
(0.0367)\end{array}$ & - & 20,241 \\
\hline 13 Emerging Markets & $\begin{array}{c}0.017 \\
(0.004)\end{array}$ & $\begin{array}{c}0.938 \\
(0.022)\end{array}$ & 0.955 & - & $\begin{array}{c}0.3973 \\
(0.0506)\end{array}$ & 3,064 \\
\hline No Dynamics & - & - & - & - & $\begin{array}{c}0.3815 \\
(0.0386)\end{array}$ & 2,938 \\
\hline All 29 Markets & $\begin{array}{c}0.021 \\
(0.004)\end{array}$ & $\begin{array}{c}0.962 \\
(0.017)\end{array}$ & 0.983 & $\begin{array}{c}0.2764 \\
(0.1024)\end{array}$ & $\begin{array}{c}0.2797 \\
(0.1193)\end{array}$ & 35,574 \\
\hline No Dynamics & - & - & - & $\begin{array}{c}0.2797 \\
(0.0423)\end{array}$ & $\begin{array}{c}0.2834 \\
(0.0648)\end{array}$ & 33,213 \\
\hline & \multicolumn{6}{|c|}{ C: Weekly IFCI Returns, July 21, 1995 to June 12, 2009} \\
\hline 16 Developed Markets & $\begin{array}{c}0.034 \\
(0.007)\end{array}$ & $\begin{array}{c}0.929 \\
(0.023)\end{array}$ & 0.963 & $\begin{array}{c}0.3032 \\
(0.1163)\end{array}$ & - & 20,741 \\
\hline No Dynamics & - & - & - & $\begin{array}{c}0.3097 \\
(0.0774)\end{array}$ & - & 19,977 \\
\hline 17 Emerging Markets & $\begin{array}{c}0.024 \\
(0.006)\end{array}$ & $\begin{array}{c}0.911 \\
(0.025)\end{array}$ & 0.935 & - & $\begin{array}{c}0.3093 \\
(0.0542)\end{array}$ & 8,643 \\
\hline No Dynamics & - & - & - & - & $\begin{array}{c}0.3019 \\
(0.0456)\end{array}$ & 8,386 \\
\hline All 33 Markets & $\begin{array}{c}0.023 \\
(0.005)\end{array}$ & $\begin{array}{c}0.940 \\
(0.018)\end{array}$ & 0.962 & $\begin{array}{c}0.4502 \\
(0.0423)\end{array}$ & $\begin{array}{c}0.3771 \\
(0.0356)\end{array}$ & 50,291 \\
\hline No Dynamics & - & - & - & $\begin{array}{c}0.4142 \\
(0.1688)\end{array}$ & $\begin{array}{c}0.4351 \\
(0.1326)\end{array}$ & 47,218 \\
\hline
\end{tabular}

Notes to Table: We report parameter estimates for the DNC models for the 16 developed markets, 13 emerging markets (IFCG), 17 emerging markets (IFCI) and all markets for different sample periods. We also report the special case of constant copula dynamic correlation $\left(\beta_{\Gamma}=0\right.$ and $\left.\alpha_{\Gamma}=0\right)$. The standard errors in parentheses are computed using the method in Engle, Shephard and Sheppard (2008). 
Table 6: Correlation Regressions. Developed Markets (DMs) and Emerging Markets (EMs). 1995-2008

Panel A: Log of Average Quarterly Panel B: Log of Average Quarterly Panel C: Log of Average Quarterly EM DM Correlation With Other DMs
Panel C: Log of Average Quarterts
Correlation With DMs
Panel D: Log of Average Quarterly EM

Correlation With Other EMs

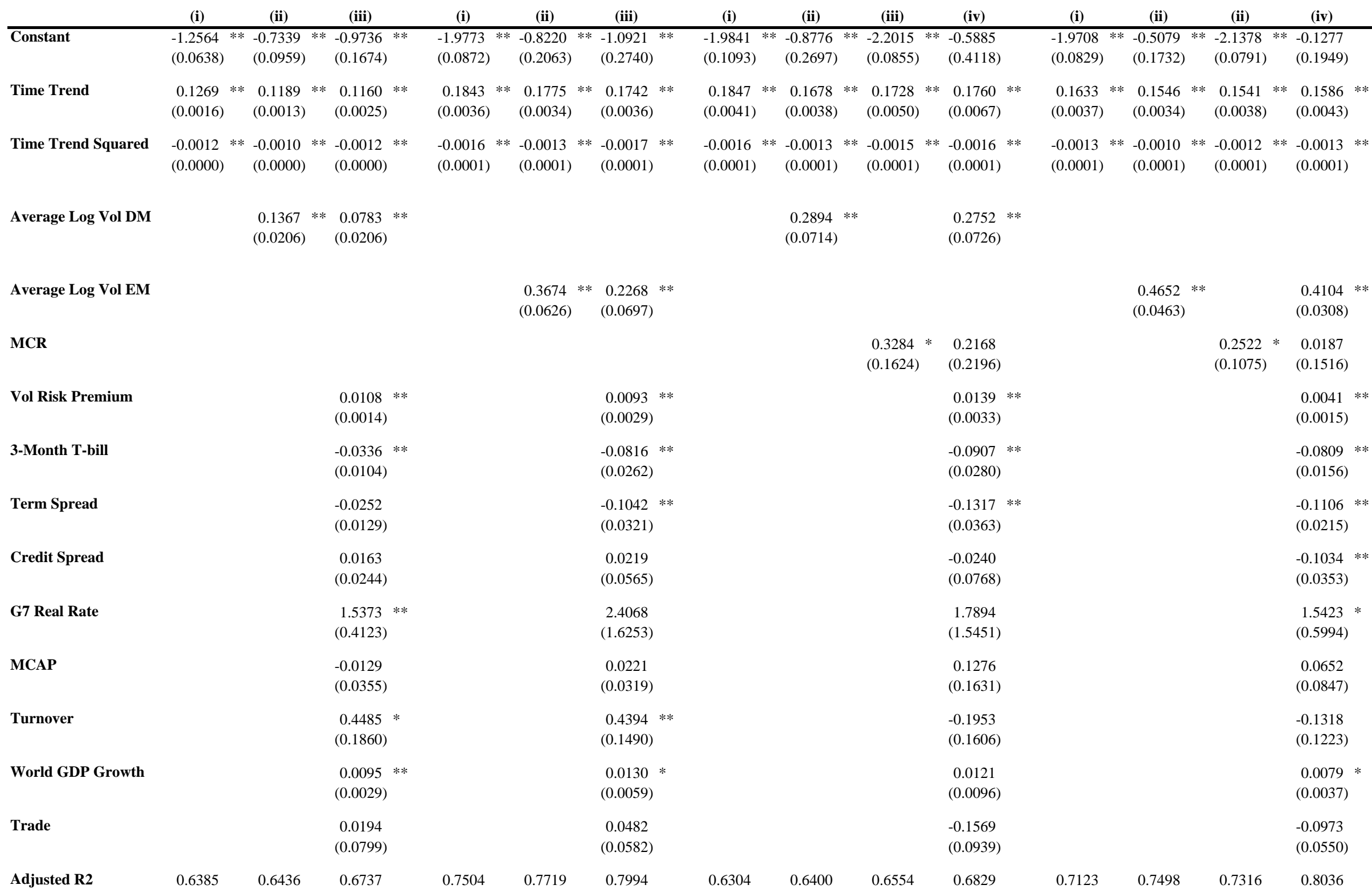

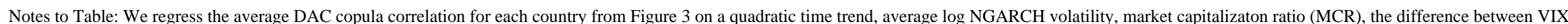

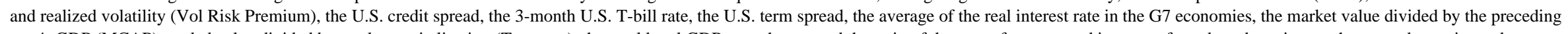
year's GDP (MCAP), traded value divided by market capitalization (Turnover), the world real GDP growth rate, and the ratio of the sum of exports and imports of goods and services to the gross domestic product

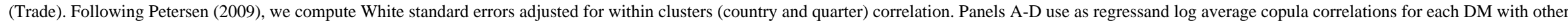
DMs, each DM with EMs, each EM with DMs, and each DM with DMs, respectively. Significance at the 5\% and $1 \%$ levels is denoted by * and **. 
2012-31: $\quad$ Eric Hillebrand, Marcelo C. Medeiros and Junyue Xu: Asymptotic Theory for Regressions with Smoothly Changing Parameters

2012-32: $\quad$ Olaf Posch and Andreas Schrimpf: Risk of Rare Disasters, Euler Equation Errors and the Performance of the C-CAPM

2012-33: Charlotte Christiansen: Integration of European Bond Markets

2012-34: Nektarios Aslanidis and Charlotte Christiansen: Quantiles of the Realized Stock-Bond Correlation and Links to the Macroeconomy

2012-35: Daniela Osterrieder and Peter C. Schotman: The Volatility of Long-term Bond Returns: Persistent Interest Shocks and Time-varying Risk Premiums

2012-36: $\quad$ Giuseppe Cavaliere, Anders Rahbek and A.M.Robert Taylor: Bootstrap Determination of the Co-integration Rank in Heteroskedastic VAR Models

2012-37: $\quad$ Marcelo C. Medeiros and Eduardo F. Mendes: Estimating High-Dimensional Time Series Models

2012-38: $\quad$ Anders Bredahl Kock and Laurent A.F. Callot: Oracle Efficient Estimation and Forecasting with the Adaptive LASSO and the Adaptive Group LASSO in Vector Autoregressions

2012-39: $\quad$ H. Peter Boswijk, Michael Jansson and Morten Ørregaard Nielsen: Improved Likelihood Ratio Tests for Cointegration Rank in the VAR Model

2012-40: $\quad$ Mark Podolskij, Christian Schmidt and Johanna Fasciati Ziegel: Limit theorems for non-degenerate $\mathrm{U}$-statistics of continuous semimartingales

2012-41: $\quad$ Eric Hillebrand, Tae-Hwy Lee and Marcelo C. Medeiros: Let's Do It Again: Bagging Equity Premium Predictors

2012-42: $\quad$ Stig V. Møller and Jesper Rangvid: End-of-the-year economic growth and time-varying expected returns

2012-43: $\quad$ Peter Reinhard Hansen and Allan Timmermann: Choice of Sample Split in Out-of-Sample Forecast Evaluation

2012-44: $\quad$ Peter Reinhard Hansen and Zhuo Huang: Exponential GARCH Modeling with Realized Measures of Volatility Statistics

2012-45: Peter Reinhard Hansen and Allan Timmermann: Equivalence Between Out-ofSample Forecast Comparisons and Wald

2012-46: $\quad$ Søren Johansen, Marco Riani and Anthony C. Atkinson: The Selection of ARIMA Models with or without Regressors

2012-47: Søren Johansen and Morten Ørregaard Nielsen: The role of initial values in nonstationary fractional time series models

2012-48: $\quad$ Peter Christoffersen, Vihang Errunza, Kris Jacobs and Hugues Langlois: Is the Potential for International Diversincation Disappearing? A Dynamic Copula Approach 